\title{
NONHYPERBOLIC PERSISTENT ATTRACTORS NEAR THE MORSE-SMALE BOUNDARY
}

\author{
Eduardo M. MUÑOZ MORALES ${ }^{*}, 1$, \\ Bernardo SAN MARTÍN REBOLLEDO ${ }^{2}$, \\ Jaime A. VERA VALENZUELA ${ }^{2}$
}

Departamento de Matemáticas, Universidad Católica del Norte, Casilla 1280, Antofagasta, Chile

Received 5 May 2002, accepted 30 September 2002

Dedicated to Professor Jacob Palis on his sixtieth birthday

ABSTRACT. - In this paper we present a submanifold $\mathcal{N}$ of codimension two contained in the Morse-Smale regular boundary in $\mathcal{X}^{r}(M)$, the space of $\mathcal{C}^{r}$ vector fields on a 3-dimensional manifold $M$, which exhibits singular cycles, such that for generic 2-parameter families going through, it has nonhyperbolic nontrivial attractors for a set of parameters with positive Lebesgue measure.

(C) 2003 L'Association Publications de l'Institut Henri Poincaré. Published by Elsevier B.V. All rights reserved

RÉSUMÉ. - Dans cet article on construit une sous-variété $\mathcal{N}$ de codimension deux contenue dans la frontière régulière de l'ensemble des champs de vecteurs Morse-Smale de $\mathcal{X}^{r}(M)$, l'espace de champs de vecteurs de classe $C^{r}$ sur la variété de dimension trois $M$, laquelle présente des cycles singuliers tels que, toute famille générique à deux paramètres transversale à $\mathcal{N}$ possède des ensembles attractifs non hyperboliques et non triviaux pour un ensemble de paramètres dont la mesure de Lebesgue est positive.

(C) 2003 L'Association Publications de l'Institut Henri Poincaré. Published by Elsevier B.V. All rights reserved

\section{Introduction}

Morse-Smale systems constitute an open set of dynamically or structurally stable elements as classically established in Palis [12] and Palis-Smale [13].

On the other hand, singular cycles constitute a bifurcation mechanism for vector fields that, to the opposite to what happen with homoclinic tangencies in the diffeomorphisms

\footnotetext{
* Corresponding author.

E-mail addresses: emunoz@ucn.cl (E.M. Muñoz Morales), sanmarti@ucn.cl (B. San Martín Rebolledo),jvera@ucn.cl (J.A. Vera Valenzuela).

${ }^{1}$ Partially supported by Fondecyt Grant 1981241, IMPA CNPq and UCN.

${ }^{2}$ Partially supported by Fondecyt Grant 1981241 and 1000047, IMPA CNPq, ICTP, Trieste, Italia and UCN.
} 
case, the hyperbolicity from the parameter point of view, is a total Lebesgue measure phenomenom for generic unfolding of these type of vector fields, see [3,11]. These kind of cycles were studied for the first time in [7], as being part of the so called singular horseshoe, an example (on manifolds with boundary) of a structurally stable vector field with a nonhyperbolic nonwandering set.

An important ingredient to obtain these results about hyperbolicity, is the presence in the cycle of just one branch of the unstable manifold of the singularity. As we will observe later, the consideration of both branches on the cycle, plus a contractivity hyphotesis for the cycle itself it will imply that the generic unfolding exhibits nonhyperbolic nontrivial attractors which are persistent in the measure sense.

Before to give the precise statement of our result we establish the setting and concepts that we will need. Let $M$ be a compact boundaryless 3-dimensional manifold and let $\mathcal{X}^{r}(M)$ be the Banach space of $C^{r}$ vector fields on $M$. For $X \in \mathcal{X}^{r}(M), \Gamma(X)$ denotes its chain recurrent set. We say that $X \in \mathcal{X}^{r}(M)$ is simple if $\Gamma(X)$ is a union of finitely many hyperbolic critical orbits. By a critical orbit we mean an orbit that is either periodic or singular. It is easy to see that the set $S^{r}$ of simple $C^{r}$ vector fields is an open subset of $\mathcal{X}^{r}(M)$.

An orbit $\gamma$ of a vector field $X$ is transversal if $\alpha(\gamma)$ and $\omega(\gamma)$ are critical hyperbolic elements and their invariant manifolds meet transversally along $\gamma$, here $\alpha$ and $\omega$ stand for the $\alpha$ and $\omega$-limit sets, respectively. We say that $X$ is a Morse-Smale system if $X$ is simple and every orbit is transversal.

A cycle $\Lambda$ of $X \in \mathcal{X}^{r}(M)$ is a compact invariant chain recurrent set consisting of finitely many orbits whose $\alpha$ and $\omega$-limit sets are critical elements linked in a cyclical way. If the cycle contains at least one singularity, we will call it a singular cycle. If $\sigma_{i}$ and $\sigma_{j}$ are critical elements on the cycle, we write $\sigma_{i} \prec \sigma_{j}$ to mean that there is an orbit $\gamma_{i j}$ on the cycle such that $\alpha\left(\gamma_{i j}\right)=\sigma_{i}$ and $\omega\left(\gamma_{i j}\right)=\sigma_{j}$.

In this paper we are concerned with those cycles $\Lambda$ whose critical elements satisfy $\sigma_{0} \prec \sigma_{1} \prec \cdots \prec \sigma_{k}=\sigma_{0}$, where $\sigma_{0}$ is the unique singularity in the cycle, $\operatorname{dim} W^{u}\left(\sigma_{0}\right)=1$ and $W^{u}\left(\sigma_{0}\right) \subset \Lambda \cap W^{s}\left(\sigma_{1}\right)$. Furthermore, if we denote by $\gamma_{l}$ and $\gamma_{r}$ the branches of $W^{u}\left(\sigma_{0}\right)$, then $\Lambda_{r}=\Lambda \backslash\left\{\gamma_{l}\right\}$ and $\Lambda_{l}=\Lambda \backslash\left\{\gamma_{r}\right\}$ are contractive singular cycles as in [3] satisfying some extra condition. In fact, as in [3] $\Lambda_{r}$ and $\Lambda_{l}$ satisfy:

(1) The eigenvalues $-\lambda_{1}, \lambda_{2}$ and $-\lambda_{3}$ of the singularity $\sigma_{0}$ are real and satisfy $-\lambda_{3}<-\lambda_{1}<0<\lambda_{2}$ and $\lambda_{1}-\lambda_{2}>0$, i.e., the cycle is contractive. Besides, we assume the aditional condition $\beta>\alpha+1$ where $\alpha=\lambda_{1} / \lambda_{2}$ and $\beta=\lambda_{3} / \lambda_{2}$.

(2) For all $p \in \gamma_{l} \cup \gamma_{r}$ and any invariant manifold $W^{c u}$ of $X$ passing through $\sigma_{0}$ tangent to the eigenspace associated with the eigenvalues $-\lambda_{1}$ and $\lambda_{2}$ holds:

$$
T_{p}\left(W^{c u}\right)+T_{p}\left(W^{s}(\omega(p))\right)=T_{p} M .
$$

(3) There is a neighbourhood $\mathcal{U}$ of $X$ such that if $Y \in \mathcal{U}$ the continuations $\sigma_{i}(Y), 0 \leqslant$ $i \leqslant k-1$, of critical orbits $\sigma_{i}(X)$ of the cycle are well defined, the vector field $Y$ is $C^{2}$-linearizing nearby $\sigma_{0}(Y)$ and the Poincare maps of $\sigma_{i}(Y), 1 \leqslant i \leqslant k-1$, are $C^{2}$-linearizing ones.

In linearizing coordinates for $\sigma_{0}$ the flow is given by $X^{t}\left(x_{3}, x_{2}, x_{1}\right)=\left(\mathrm{e}^{-\lambda_{3} t} x_{3}\right.$, $\left.\mathrm{e}^{\lambda_{2} t} x_{2}, \mathrm{e}^{-\lambda_{1} t} x_{1}\right)$ and then $W_{\text {loc }}^{s}\left(\sigma_{0}\right)=\left\{\left(x_{3}, 0, x_{1}\right)\right\}$ and $W_{\text {loc }}^{u}\left(\sigma_{0}\right)=\left\{\left(0, x_{2}, 0\right)\right\}$. We define $\Sigma_{0}=\left\{\left(x_{3}, x_{2}, 1\right)\right\}$. 
Let $\Sigma_{1}$ be a transversal section to the flow through a point $q$ of $\sigma_{1}$, then in linearizing coordinates, $\{(x, 0)\}$ and $\{(0, y)\}$ are the local stable and unstable manifolds of the fixed point $q=(0,0)$ of the Poincare map induced by $\sigma_{1}$, respectively. The flow generated by $X$ also induces a Poincaré map $\mathcal{P}_{01}: \operatorname{Dom}\left(\mathcal{P}_{01}\right) \subset \Sigma_{0} \rightarrow \Sigma_{1} . \mathcal{P}_{01}(w)$ is the first point of intersection among the positive orbit of $w$ and the section $\Sigma_{1}$.

(4) The orbit $\gamma_{k-1,0}$ in the cycle is not contained in the strong stable manifold of $\sigma_{0}$, and we suppose that intersects $\Sigma_{0}$.

(5) The branch $\gamma_{l}$ (resp. $\gamma_{r}$ ) of $W^{u}\left(\sigma_{0}(X)\right)$ is the only nontransversal orbit in $\Lambda_{l}$ (resp. $\Lambda_{r}$ ). Moreover, if $\sigma \subset \Lambda=\Lambda_{l} \cup \Lambda_{r}$ is a periodic orbit, then there is only one regular orbit $\gamma$ in the cycle $\Lambda$ such that $\sigma=\alpha(\gamma)$.

(6) For $j=l, r, \Lambda_{j}$ is isolated, i.e., there exists a neighbourhood $U_{j}$ of $\Lambda_{j}$, which is called an isolating block such that $\bigcap_{t} X^{t}\left(U_{j}\right)$ does not contains any orbit close to $W^{u}\left(\sigma_{0}(X)\right)$. Here $X^{t}: M \hookleftarrow$ is the flow generated by $X . U=U_{l} \cup U_{r}$ is an isolating block for $\Lambda$.

In Fig. 1 a sketch of the geometrical shape of our cycle considering the coordinates system as mentioned in (3), is shown.

Let $C^{k}\left(I^{2}, \mathcal{X}^{r}(M)\right), k \geqslant 2$, be the space of families $\left\{X_{\mu, v}\right\}$ of $C^{r}$-vector fields, $r \geqslant 2$, depending on two parameters $(\mu, v) \in I^{2}=[-1,1] \times[-1,1]$ such that the map $(\mu, v) \in$ $I^{2} \rightarrow X_{\mu, v} \in \mathcal{X}^{r}(M)$ is $C^{k}$. Let us endow $C^{k}\left(I^{2}, \mathcal{X}^{r}(M)\right)$ with the $C^{k}$-topology. We are interested in families $\left\{X_{\mu, v}\right\} \in C^{k}\left(I^{2}, \mathcal{X}^{r}(M)\right)$ such that (after a reparametrization, if necessary) $X_{0,0}$ has a singular cycle as described above.

We say that a compact invariant transitive set $Q$ of a vector field $X$ is an attractor if there is a neighbourhood $U \supseteq Q$ such that $\overline{X^{t}(U)} \subseteq U$ for all $t>0$ and $Q=$ $\bigcap_{t \geqslant 0} X^{t}(U)$. If $Q$ is a transitive set but not a regular orbit, we say that it is a nontrivial attractor. Now, we state our main result:

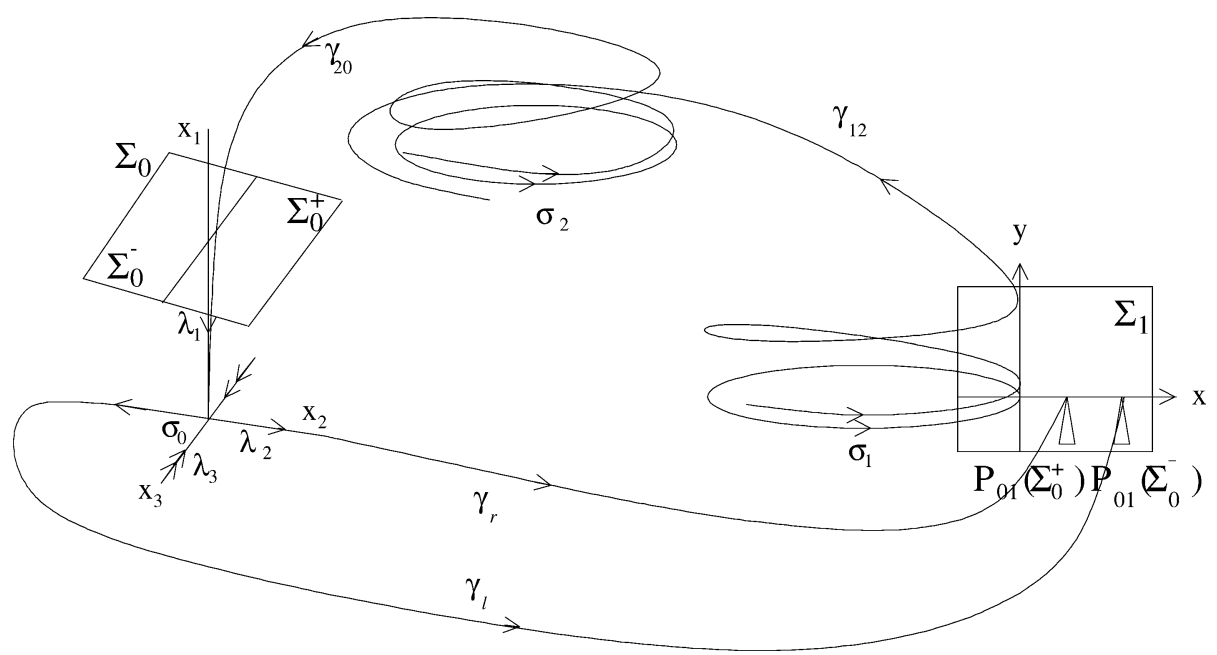

Fig. 1. 
THEOREM 1. - For generic families $\left\{X_{\mu, \nu}\right\} \in C^{k}\left(I^{2}, \mathcal{X}^{r}(M)\right)$ having a singular cycle for $X_{\left(\mu_{0}, v_{0}\right)}=X_{(0,0)}$ that satisfies the conditions (1)-(6) above, it holds that

$$
m\left(\left\{(\mu, \nu): X_{\mu, \nu} \text { has a nonhyperbolic nontrivial attractor }\right\}\right)>0
$$

where $m$ stands for the Lebesgue measure in $I^{2}$. Moreover, this set accumulates $(0,0)$.

Remark 1. -

(1) The condition $\beta>\alpha+1$ in (1) above had been established in order to obtain certain appropiate $C^{2}$-invariant foliation (Lemma 1, Section 2), leading us to a one-dimensional dynamics reduction on our study. Besides, in Lemma 3 we need this class of differentiability in order to apply Mañé's Theorem [8].

(2) Condition (2) above also means that $W^{c u}\left(\sigma_{0}\right)$ meets $W^{s}\left(\sigma_{1}\right)$ transversally.

(3) The conditions (5) and (6) are required in order to guarantee that we can construct the vector field $X$ in the boundary of the Morse-Smale systems. In fact, by condition (5) we can slightly perturb the system $X$ "moving down" the first intersection point belonging to each of both branches of $W^{u}\left(\sigma_{0}\right)$ with $\Sigma_{1}$ without create homoclinic points (this can not happen if we "move this point up"), and then the condition (6) (with some extra hypothesis about the existence of a filtration, see [18] for definitions and details) guarantee us that there are not changes ( $\Omega$-explosion) in the dynamics far away from the cycle. In this way we can attain the dynamics of $X$ (the cycle) from the Morse-Smale systems and therefore this kind of cycles constitute a bifurcation mechanism for the MorseSmale systems.

(4) Condition (6) implies that the eigenvalues associated to the $\sigma_{1}$ are positives, otherwise a singular horseshoe appears [7].

(5) Condition (5) contains the only two degeneracencies, so the cycle is a codimension two phenomena.

(6) The attractors obtained are one side orientation preserving Lorenz-like atractors.

(7) A similar result can be obtained when we consider cycles in which the branches of the singularity $\sigma_{0}$ are linked to distinct periodic orbits. This result is established in a forthcoming paper [17].

(8) The case where the cycle reduces to a double homoclinic cycle has been studied in [9].

The paper is organized as follows. To understand the dynamics of $X_{\mu, \nu}, \mu, v>0$, we restrict our analysis to the dynamics of $X_{\mu, \nu}$ on $\Gamma\left(X_{\mu, \nu}, U\right)$ where $U$ is an isolating block. In Section 2 we establish the existence of a $C^{2}$-invariant stable foliation (Lemma 1). This reduces our study to the dynamics of one-dimensional maps $f_{\mu, v}$, and some limit dynamic is obtained via renormalization (Lemma 2). In Section 3 we show that for a large enough positive integer $n$, there exists a positive Lebesgue measure set $E_{n}$ in the $(\mu, v)$-parameter space such that the critical values of $g_{\mu, \nu}=\left(f_{\mu, \nu}\right)^{n+1}$ have positive Lyapunov exponents and the clousure of the $g_{\mu, \nu}$-iterates of these points is an interval (Theorem 2). From this result our Theorem 1 follows. Theorem 2 is proved by applying Benedicks-Carleson techniques, and in order to do that, a fundamental and crucial fact is to prove that the maximal orbits outside of a certain neighbourhood of 
the critical points have exponential growth, provided that the number of elements in the orbit is big enough.

\section{Renormalization}

Our first proposition, Proposition 1, points out that systems having our cycles constitute (locally) submanifolds of codimension two in $\mathcal{X}^{r}(M)$ contained in the boundary of the Morse-Smale systems and, consequently, the natural approach to study their bifurcations is by mean of two parameter families transversal to these codimension two submanifolds.

PROPOSITION 1. - Let $\Lambda$ be a singular cycle for the vector field $X$ as described in the introduction, and let $U$ be an isolating block associated to it. Then, there exists a neighbourhood $\mathcal{U}$ of $X$ in $\mathcal{X}^{r}(M)$ and a $C^{r}$-submanifold $\mathcal{N}$ of codimension two in $\mathcal{X}^{r}(M)$ containing $X$ such that if $Y \in \mathcal{N} \cap \mathcal{U}$, then $\Gamma(Y, U)=\bigcap_{t} Y^{t}(U)$ contains a cycle topologically equivalent to $\Lambda$.

Proof. - The proof is quite simple and it goes as follows: let $\gamma_{r}$ and $\gamma_{l}$ be the branches of $W^{u}\left(\sigma_{0}\right)$. Let $\Sigma_{1}$ be a transversal section to $X$ at $q \in \sigma_{1}$. For each $Y$ close to $X$ we consider linearizing coordinates $(x, y)$ on $\Sigma_{1}$ for the Poincaré map associated to $\sigma_{1}$ (which vary smoothly with $Y$ ) such that $W_{\text {loc }}^{s}\left(\sigma_{1}\right) \cap \Sigma_{1}=\{(x, 0)\}$. If $\mathcal{U}$ is a small enough neighbourhood of $X$, then we can define the $C^{r}$-map $G_{i}: \mathcal{U} \rightarrow \mathbb{R}$ by $G_{i}(Y)=\pi_{2} \circ p_{i}(Y)$, where $p_{i}(Y)$ is the first intersection of $\gamma_{i}$ with $\Sigma_{1}, i=l, r$, and $\pi_{2}$ denotes the projection over the second term on the coordinates of $\Sigma_{1}$. It is clear that $B_{r}=G_{r}^{-1}(0)$ is a submanifold of codimension one of $\mathcal{U}$ that contains $X$. Analogously we obtain $B_{l}=G_{l}^{-1}(0)$ which is transversal to $B_{r}$, and thus we get that both, $\mathcal{N}=B_{r} \cap B_{l}$ and $\mathcal{U}$, satisfy the conditions of the proposition. In fact, if $G: \mathcal{U} \rightarrow \mathbb{R}^{2}$ is defined by $G(Y)=\left(G_{r}(Y), G_{l}(Y)\right)$ then $G$ is a submersion and $\mathcal{N}=G^{-1}(0,0)$.

Let $X$ be a vector field having a cycle $\Lambda$ as above, and let $S$ be a two-dimensional $C^{k}$-submanifold of $\mathcal{X}^{r}(M)$ transversal to $\mathcal{N}$ at $X$. The map $G: S \rightarrow \mathbb{R}^{2}$ defined by $G(Y)=\left(G_{r}(Y), G_{l}(Y)\right)$ is a $C^{k}$-diffeomorphism in a neighbourhood of $X$ in $S$ and then, it determines a parametrization of $S$ in a neighbourhood of $X$, i.e., $Y=X_{\mu, v}$ if and only if $G(Y)=(\mu, v)$. Moreover, $X=X_{0,0}$ and $X_{\mu, \nu}$ is a Morse-Smale system if and only if $\mu$ and $v$ are both negatives. Furthermore, from $[3,6,11,16]$ we know that in the Lebesgue sense, for almost every $(\mu, \nu)$ close enough to $(0,0)$ and such that $\mu \nu<0$ we have that $X_{\mu, v}$ is an Axiom $A$ system. Therefore, our work will be focus on the region corresponding to positive values for the parameters $\mu$ and $v$.

Let $\left\{X_{\mu, v}\right\} \in C^{k}\left(I^{2}, \mathcal{X}^{r}(M)\right)$, and let $\Lambda$ be a singular cycle associated to the vector field $X=X_{0,0}$. Using transversality arguments we can change the cycle for other one, choosing a new cycle contained in $\Gamma(X, U)$, still named $\Lambda$, having only one periodic orbit, i.e., $\Lambda=\left\{\sigma_{0}, \gamma_{r}, \gamma_{l}, \sigma_{1}\right\}$ linked by $\sigma_{0} \prec \sigma_{1} \prec \sigma_{2}=\sigma_{0}$.

Let $\Sigma_{1}$ be a cross section to the flow of $X$ at $q \in \sigma_{1}(X)$ and choose $C^{2}$-linearizing coordinates $(x, y)$ for the Poincaré map induced by $\sigma_{1}\left(X_{\mu, v}\right)$, depending smoothly on the parameters. In this coordinates we have: 
(1) $Q=\{(x, y) /|x|,|y| \leqslant 2\} \subset \Sigma_{1}$.

(2) $\{(x, 0) /|x| \leqslant 1\} \subset W^{s}\left(\sigma_{1}\left(X_{\mu, \nu}\right)\right)$.

(3) $\{(0, y) /|y| \leqslant 1\} \subset W^{u}\left(\sigma_{1}\left(X_{\mu, v}\right)\right)$.

(4) $\Gamma\left(X_{\mu, v}, U\right) \cap \Sigma_{1} \subset\{(x, y) / y \geqslant 0\}$.

(5) The Poincaré map $P_{\mu, \nu}$, defined by $\sigma_{1}\left(X_{\mu, \nu}\right)$ is linear and $P_{\mu, \nu}(x, y)=$ $\left(\tau_{\mu, \nu} x, \rho_{\mu, \nu} y\right)$ where $0<\tau_{\mu, \nu}<1$ and $\rho_{\mu, \nu}>1$ (see (5) and (6) in Section 1).

A closed subset $R \subset \Sigma_{1}$ is called a horizontal strip if it is bounded (in $Q$ ) by two disjoint continuous curves that connect the vertical sides of $Q$.

There are two horizontal strips $R_{0}$ and $R_{1}$ in $Q$ such that for $(\mu, v)$ close to $(0,0)$, the $X_{\mu, \nu}$ positive orbit of any point in $R_{0} \cup R_{1}$ meets $\Sigma_{1}$ and then, there is a $(\mu, v)$-dependent first return map $F_{\mu, v}: R_{0} \cup R_{1} \rightarrow \Sigma_{1}$. In fact, $R_{0}$ is a horizontal strip containing the $P_{\mu, v^{-}}$ local stable manifold of $q_{\mu, \nu}$, and $F_{\mu, \nu}$ restricted to this strip coincides with the Poincaré map $P_{\mu, \nu} . R_{1}$ is a horizontal strip containing in its interior the connected component $W_{0,0}^{s}$ of $W^{s}\left(\sigma_{0}\left(X_{0,0}\right)\right) \cap Q$ which contains the last intersection point among $\gamma_{1,0}$ (time oriented) and $Q$. Here we note that, as in [3] the strip $R_{1}$, there denoted by $R_{Y}^{0}$, depends on each vector field $Y=X_{\mu, \nu}$ close to $X$. In [3] one of the horizontal sides of $R_{Y}^{0}$ is contained in the continuous variation $W_{\mu, \nu}^{s}$ of $W_{0,0}^{s}$ with respect to the vector field $X_{\mu, \nu}$. In that situation, the return map is defined only at one side of $W_{\mu, v}^{s}$, whereas in our case, the return map is defined at both sides of $W_{\mu, v}^{s} \subset R_{1}$ (this fact introduces a discontinuity in the Poincaré return map). In fact, the positive orbit of a point in $R_{1}$ has a segment close to one of the branches $\gamma_{l}$ or $\gamma_{r}$ of $W^{u}\left(\sigma_{0}\left(X_{\mu, \nu}\right)\right)$ and then intersects $\Sigma_{1}$, as it is shown in Fig. 2.

For simplicity, we observe that after multiplication by $(\mu, v)$-dependent constants in the coordinates we can suppose $W_{\mu, \nu}^{s} \cap\{(0, y)\}=\{(0,1)\}$.

In order to find some analytical expresion for $F_{\mu, \nu}$ we choose $\left(x_{3}, x_{2}, x_{1}\right) C^{2}$ linearizing coordinates for the singularity $\sigma_{0}$ depending smoothly on the vector field. In these coordinates the $X_{\mu, \nu}$ flow is given by $X_{\mu, v}^{t}\left(x_{3}, x_{2}, x_{1}\right)=\left(\mathrm{e}^{-\lambda_{3} t} x_{3}, \mathrm{e}^{\lambda_{2} t} x_{2}, \mathrm{e}^{-\lambda_{1} t} x_{1}\right)$ where $-\lambda_{3}<-\lambda_{1}<0<\lambda_{2}$ are the $(\mu, v)$-dependent eingenvalues of $D X_{\mu, v}\left(\sigma_{0}\left(X_{\mu, \nu}\right)\right)$.

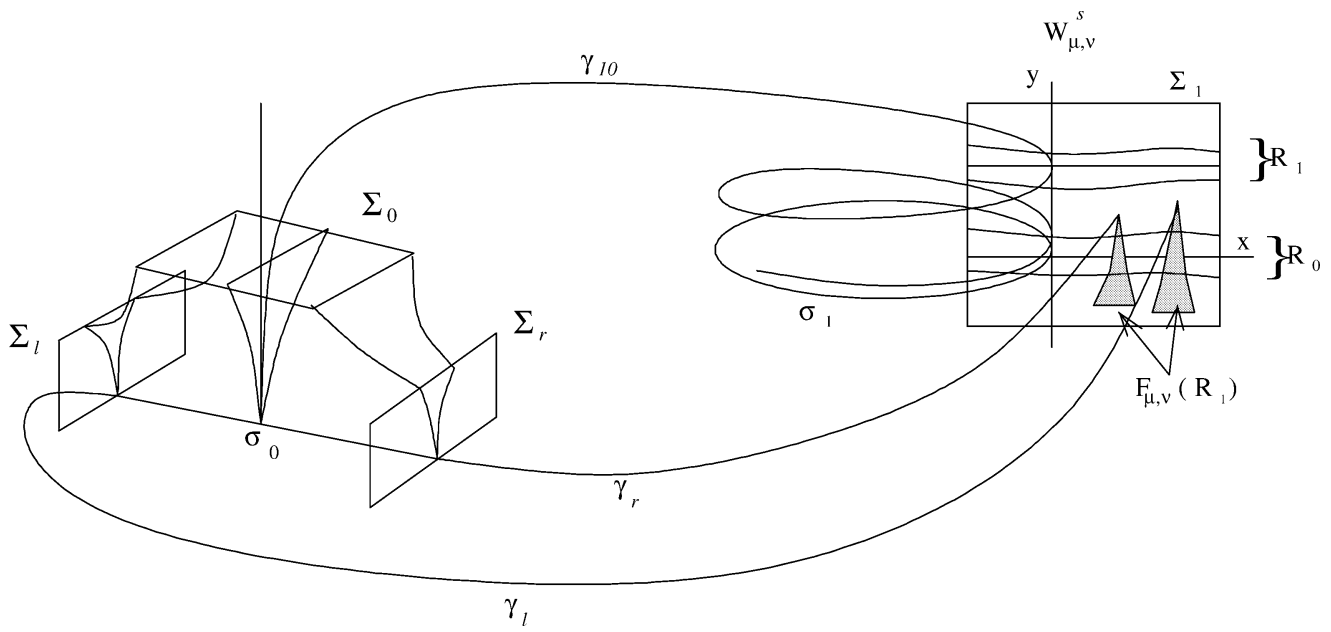

Fig. 2. 
Next, we take the sections $\Sigma_{0}=\left\{\left(x_{3}, x_{2}, 1\right)\right\}, \Sigma_{l}=\left\{\left(x_{3},-1, x_{1}\right)\right\}$ and $\Sigma_{r}=\left\{\left(x_{3}, 1\right.\right.$, $\left.\left.x_{1}\right)\right\}$ (see Fig. 2), and consider the ( $\left.\mu, v\right)$-dependent Poincaré map $P_{1}: \Sigma_{0} \backslash\left\{\left(x_{3}, 0,1\right)\right\} \rightarrow$ $\Sigma_{l} \cup \Sigma_{r}$ induced by the $X_{\mu, \nu}$ flow given by $P_{1}\left(x_{3}, x_{2}, x_{1}\right)=\left(x_{3}\left|x_{2}\right|^{\beta_{\mu, v}},\left|x_{2}\right| / x_{2},\left|x_{2}\right|^{\alpha_{\mu, v}}\right)$, where $\alpha_{\mu, v}=\lambda_{1} / \lambda_{2}$ and $\beta_{\mu, v}=\lambda_{3} / \lambda_{2}$.

Finally, we consider the $(\mu, \nu)$-dependent diffeomorphisms $P_{0}: \mathcal{D o m}\left(P_{0}\right) \subset \Sigma_{1} \rightarrow \Sigma_{0}$ and $P_{2}: \operatorname{Dom}\left(P_{2}\right) \subset \Sigma_{l} \cup \Sigma_{r} \rightarrow \Sigma_{1}$ induced by the $X_{\mu, v}$ flow. Then, for $F_{\mu, v}$ restricted to $R_{1}$ we have

$$
F_{\mu, v}(x, y)=\left(P_{2} \circ P_{1} \circ P_{0}\right)(x, y)=\left(u_{\mu, v}(x, y), v_{\mu, v}(x, y)\right) .
$$

By using the chain rule it is inmediate that there is a positive constant $C$ independent from the parameter $(\mu, v)$ such that

$$
\begin{aligned}
& C^{-1}\left|x_{2}\right|^{\alpha_{\mu, v}-1} \leqslant\left|\partial_{y} v_{\mu, v}(x, y)\right| \leqslant C\left|x_{2}\right|^{\alpha_{\mu, v}-1}, \\
& C^{-1}\left|x_{2}\right|^{\alpha_{\mu, v}-2} \leqslant\left|\partial_{y}^{2} v_{\mu, v}(x, y)\right| \leqslant C\left|x_{2}\right|^{\alpha_{\mu, v}-2} .
\end{aligned}
$$

The following lemma, whose proof is obtained in the same way than in Lemma 1 of [3] (see also p. 695 in [7]), implies that after a $C^{2}$-change of coordinates the second component of $F_{\mu, \nu}$ does not depend on $x$, i.e., $v_{\mu, v}(x, y)=f_{\mu, \nu}(y)$ and therefore, our study is reduced to a setting in one-dimensional dynamics.

LEMMA 1. - If $\beta>\alpha+1$ then for each pair $(\mu, v)$ small enough there exists a $C^{2}$ invariant stable foliation $\mathcal{F}_{\mu, v}^{s}$ defined on $\Sigma_{1}$ with a $C^{k}$-dependence on the parameter $(\mu, v)$.

Using this lemma, the formula for $P_{1}$ and Taylor expantion for $P_{0}$ and $P_{2}$, we have that after a $C^{2}$-change of coordinates given by proyection along the leaves of $\mathcal{F}_{\mu, \nu}^{s}$, and depending $C^{k}$ on the parameter $(\mu, \nu)$, the return map $F_{\mu, \nu}$ can be expresed by

$$
F_{\mu, v}(x, y)= \begin{cases}\left(\widetilde{\mu}_{\mu, v}(x, y), f_{\mu, v}(y)\right), & (x, y) \in R_{1}, \\ \left(\tau_{\mu, v} x, f_{\mu, v}(y)\right), & (x, y) \in R_{0},\end{cases}
$$

where $\left\{f_{\mu, \nu}\right\}$ is the $C^{k}$-two parameter family of one-dimensional $C^{2}$-maps induced by the stable foliation $\mathcal{F}_{\mu, \nu}^{s}$ defined by

$$
f_{\mu, \nu}(y)= \begin{cases}\rho_{\mu, \nu} y, & 0 \leqslant y \leqslant \rho_{\mu, v}^{-1} b(\mu, v), \\ \mu-k_{\mu, \nu}(y)(1-y)^{\alpha_{\mu, \nu},}, & a(\mu, v) \leqslant y<1, \\ v-\tilde{k}_{\mu, \nu}(y)(y-1)^{\alpha_{\mu, \nu},} & 1<y \leqslant b(\mu, v) .\end{cases}
$$

The functions $(\mu, v, y) \rightarrow k_{\mu, v}(y)$ and $(\mu, v, y) \rightarrow \tilde{k}_{\mu, v}(y)$ are positives, bounded and bounded away from zero (see (1)) and besides, $C^{k}$ on the parameter $(\mu, v)$ and $C^{2}$ on $y \in[a(\mu, \nu), 1) \cup(1, b(\mu, \nu)]$. The extreme points $a=a(\mu, \nu)$ and $b=b(\mu, \nu)$ are defined by the relations

$$
\mu-k_{\mu, \nu}(a)(1-a)^{\alpha_{\mu, v}}=0 \quad \text { and } \quad v-\tilde{k}_{\mu, v}(b)(b-1)^{\alpha_{\mu, \nu}}=0 .
$$

Note that this relation is given to leave out those values of $y$ such that $f_{\mu, v}(y)<0$, because they are not related to $\Gamma\left(X_{\mu, v}, U\right)$, since their orbits come back to $\Sigma_{1}$ at most a finite number of times, see Fig. 3. 


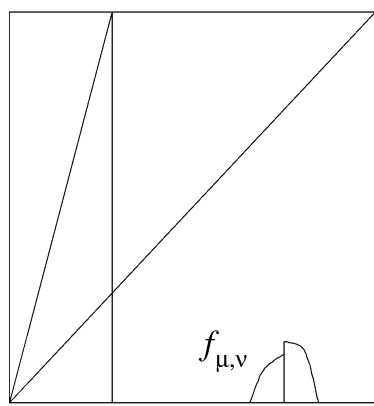

$\mu, v<2 p_{\mu, v}^{-\mathrm{n}}$

(a)

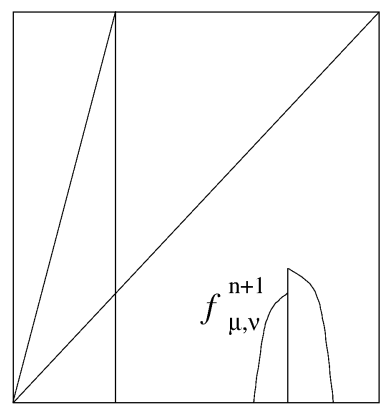

$\rho_{\mu, v}^{\mathrm{n}} \mu, \rho_{\mu, v}^{\mathrm{n}} \nu<1$

(b)

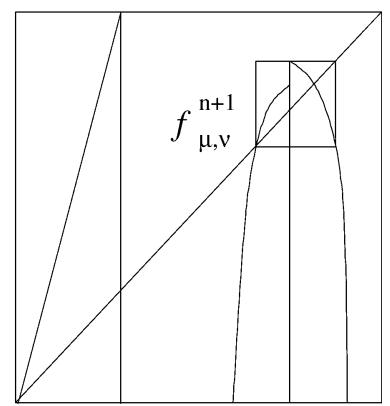

$1<\rho_{\mu, v}^{\mathrm{n}} \mu, \rho_{\mu, \nu}^{\mathrm{n}} \nu<\mathrm{b}$

(c)

Fig. 3.

Furthermore, from (1) we conclude that there are positive constants $K_{1}$ and $K_{2}$, independents from the parameter $(\mu, \nu)$ such that

$$
\begin{aligned}
& K_{1}|1-y|^{\alpha_{\mu, v}-1}<\left|\partial_{y} f_{\mu, \nu}(y)\right|<K_{2}|1-y|^{\alpha_{\mu, v}-1}, \\
& K_{1}|1-y|^{\alpha_{\mu, \nu}-2}<\left|\partial_{y}^{2} f_{\mu, v}(y)\right|<K_{2}|1-y|^{\alpha_{\mu, \nu}-2} .
\end{aligned}
$$

For a positive integer $n$, consider $g_{n, \mu, \nu}=\left.\left(f_{\mu, \nu}^{n+1}\right)\right|_{[a, b] \backslash\{1\}}$ where $a=a(\mu, \nu)$ and $b=b(\mu, v)$. Note that $g_{n, \mu, v}$ may be not defined for every $y \in[a, b] \backslash\{1\}$. However, if we suppose $\mu \leqslant \rho_{\mu, \nu}^{-n} a$ and $\nu \leqslant \rho_{\mu, \nu}^{-n} b$ then a straightforward evaluation gives

$$
g_{n, \mu, v}(y)= \begin{cases}\rho_{\mu, \nu}^{n}\left[\mu-k_{\mu, \nu}(y)(1-y)^{\alpha_{\mu, \nu}}\right], & a \leqslant y<1, \\ \rho_{\mu, \nu}^{n}\left[v-\tilde{k}_{\mu, \nu}(y)(y-1)^{\alpha_{\mu, \nu}}\right], & 1<y \leqslant b .\end{cases}
$$

In Fig. 3(a), (b) and (c), we have been drawn the graphs of $f_{\mu, \nu} ; f_{\mu, \nu}^{n+1}$ for $\rho_{\mu, \nu}^{n} \mu<1$ and $\rho_{\mu, \nu}^{n} v<1$; and the graph of $f_{\mu, \nu}^{n+1}$ for $1<\rho_{\mu, \nu}^{n} \mu<b$ and $1<\rho_{\mu, v}^{n} v<b$. In the next section our attention will be focus on the case sketched in Fig. 3(c).

The next lemma is similar to Theorem 1, p. 47 in [14], and it means that after a suitable $n$-dependent reparametrization there is a well defined limit dynamic for $g_{n}$ when $n$ converges to infinite. These reparametrizations will be done in neighbourhoods of some special parameters values $\mu_{n}^{\prime}$ and $v_{n}^{\prime}$. In order to do that, we have the following:

CLAIM 1. - Given $n$ big enough, there are unique parameters $\mu_{n}^{\prime}$ and $v_{n}^{\prime}$ such that $\mu_{n}^{\prime}$ $=v_{n}^{\prime}$ and $\rho_{\mu_{n}^{\prime}, v_{n}^{\prime}}^{n} \mu_{n}^{\prime}=1$ hold.

Proof. - For $\mu$ such that $\rho_{\mu, \mu}^{n} \mu<2$, let $w_{n}(\mu)=\rho_{\mu, \mu}^{n} \mu$. Taking derivative we obtain, for some constant,

$$
\partial_{\mu} w_{n}(\mu)=\rho_{\mu, \mu}^{n}\left[n \rho_{\mu, \mu}^{-1} \partial_{\mu}\left(\rho_{\mu, \mu}\right) \mu+1\right] \geqslant C \rho_{\mu, \mu}^{n}
$$

and then $w_{n}(\mu)$ is an increasing function of $\mu$. Consequently, there is a unique $\mu=\mu_{n}^{\prime}$ such that $\rho_{\mu_{n}^{\prime}, \mu_{n}^{\prime}}^{n} \mu_{n}^{\prime}=1$. 
Lemma 2 (Renormalization). - For $\alpha=\alpha_{0,0}=\lambda_{1} / \lambda_{2}$ and $K=\tilde{k}_{0,0}(1) / k_{0,0}(1)$ consider the two-parameter family

$$
h_{\bar{\mu}, \bar{v}}(\bar{y})= \begin{cases}-(-\bar{y})^{\alpha}+\bar{\mu}, & \bar{y}<0, \\ -K(\bar{y})^{\alpha}+\bar{v}, & 0<\bar{y} .\end{cases}
$$

Define $F(y, \mu, v)=\left(f_{\mu, v}(y), \mu, v\right)$, and $\Phi(\bar{y}, \bar{\mu}, \bar{v})=\left(h_{\bar{\mu}, \bar{v}}(\bar{y}), \bar{\mu}, \bar{v}\right)$. Then, for each positive integer $n$, there are $n$-dependent reparametrizations $\mu=\mu_{n}(\bar{\mu}, \bar{v}), v=v_{n}(\bar{\mu}, \bar{v})$ of the $(\mu, \nu)$-parameter, and $(\bar{\mu}, \bar{v})$-dependent changing coordinate transformations $\psi_{n, \bar{\mu}, \bar{v}}$ such that if we consider the functions

$$
\begin{aligned}
& \phi_{n}(\bar{y}, \bar{\mu}, \bar{v})=\left(\psi_{n, \bar{\mu}, \bar{v}}(\bar{y}), \mu_{n}(\bar{\mu}, \bar{v}), v_{n}(\bar{\mu}, \bar{v})\right), \\
& \Phi_{n}=\left(\phi_{n}\right)^{-1} \circ F^{n+1} \circ \phi_{n},
\end{aligned}
$$

then $\Phi_{n}(\bar{y}, \bar{\mu}, \bar{v})$ converges $C^{2}$ to $\Phi(\bar{y}, \bar{\mu}, \bar{v})$ on any compact set of $\mathbb{R} \backslash\{0\} \times \mathbb{R}^{2}$. Moreover, the $\phi_{n}$-image of a compact set $K \subset \mathbb{R}^{3}$, converges, for $n \rightarrow \infty$, in the $(y, \mu, v)$ space to $(1,0,0)$. Furthermore, if $h_{n, \bar{\mu}, \bar{v}}=\left(\psi_{n, \bar{\mu}, \bar{v}}\right)^{-1} \circ g_{n, \mu, \nu} \circ \psi_{n, \bar{\mu}, \bar{v}}$ then there are positive constants $K_{1}$ and $K_{2}$ independent of either $n \in \mathbb{N}$ and the $\bar{\mu}, \bar{v}$ parameters, such that

$$
\begin{aligned}
& K_{1}|\bar{y}|^{\alpha_{\mu, v}-1}<\left|\partial_{\bar{y}} h_{n, \bar{\mu}, \bar{v}}(\bar{y})\right|<K_{2}|\bar{y}|^{\alpha_{\mu, v}-1}, \\
& K_{1}|\bar{y}|^{\alpha_{\mu, v}-2}<\left|\partial_{\bar{y}}^{2} h_{n, \bar{\mu}, \bar{v}}(\bar{y})\right|<K_{2}|\bar{y}|^{\alpha_{\mu, \nu}-2} .
\end{aligned}
$$

Proof. - Take $n \in \mathbb{N}$. Given $\mu, v>0$ define

$$
c_{n}(\mu, \nu)=\left(k_{0,0}(1) \rho_{\mu, \nu}^{n}\right)^{\frac{1}{1-\alpha_{\mu, \nu}}}, \quad c_{n}=c_{n}(\mu, \nu)>0 .
$$

Since $\alpha_{\mu, v}>1$ we have

$$
\rho_{\mu, \nu}^{n} c_{n}^{\alpha_{\mu, v}-1}=\frac{1}{k_{0,0}(1)} \quad \text { and } \quad c_{n} \rightarrow 0 \quad \text { as } n \rightarrow \infty .
$$

Now, define

$$
\bar{\mu}=c_{n}^{-1}\left(\rho_{\mu, \nu}^{n} \mu-1\right) \quad \text { and } \quad \bar{v}=c_{n}^{-1}\left(\rho_{\mu, \nu}^{n} v-1\right) .
$$

A straightforward computation give us

$$
\begin{aligned}
& \partial_{\mu} \bar{\mu}=c_{n}^{-2}\left[\left(n \rho_{\mu, \nu}^{n-1} \partial_{\mu}\left(\rho_{\mu, \nu}\right) \mu+\rho_{\mu, \nu}^{n}\right) c_{n}-\left(\rho_{\mu, \nu}^{n} \mu-1\right) \partial_{\mu} c_{n}\right], \\
& \partial_{\nu} \bar{\mu}=c_{n}^{-2}\left[n \rho_{\mu, \nu}^{n-1} \partial_{\nu}\left(\rho_{\mu, \nu}\right) \nu c_{n}-\left(\rho_{\mu, \nu}^{n} v-1\right) \partial_{\nu} c_{n}\right], \\
& \partial_{\mu} \bar{\nu}=c_{n}^{-2}\left[n \rho_{\mu, \nu}^{n-1} \partial_{\mu}\left(\rho_{\mu, \nu}\right) \nu c_{n}-\left(\rho_{\mu, \nu}^{n} \nu-1\right) \partial_{\mu} c_{n}\right], \\
& \partial_{\nu} \bar{\nu}=c_{n}^{-2}\left[\left(n \rho_{\mu, \nu}^{n-1} \partial_{\nu}\left(\rho_{\mu, \nu}\right) \nu+\rho_{\mu, \nu}^{n}\right) c_{n}-\left(\rho_{\mu, \nu}^{n} \nu-1\right) \partial_{\nu} c_{n}\right],
\end{aligned}
$$

and taking $\mu_{n}^{\prime}$ and $v_{n}^{\prime}$ as in the claim 1, then for $\frac{\partial(\bar{\mu}, \bar{v})}{\partial(\mu, v)}=\frac{\partial(\bar{\mu}, \bar{v})}{\partial(\mu, v)}\left(\mu_{n}^{\prime}, v_{n}^{\prime}\right)$ we obtain 


$$
\frac{\partial(\bar{\mu}, \bar{v})}{\partial(\mu, v)}=\frac{1}{c_{n}\left(\mu_{n}^{\prime}, v_{n}^{\prime}\right)}\left[\begin{array}{cc}
n \rho_{\mu_{n}^{\prime}, v_{n}^{\prime}}^{-1} \partial_{\mu}\left(\rho_{\mu, v}\right)+\rho_{\mu_{n}^{\prime}, v_{n}^{\prime}}^{n} & n \rho_{\mu_{n}^{\prime}, v_{n}^{\prime}}^{-1} \partial_{\nu}\left(\rho_{\mu, v}\right) \\
n \rho_{\mu_{n}^{\prime}, v_{n}^{\prime}}^{-1} \partial_{\mu}\left(\rho_{\mu, v}\right) & n \rho_{\mu_{n}^{\prime}, v_{n}^{\prime}}^{-1} \partial_{\nu}\left(\rho_{\mu, v}\right)+\rho_{\mu_{n}^{\prime}, v_{n}^{\prime}}^{n}
\end{array}\right] .
$$

From here

$$
\operatorname{det}\left[\frac{\partial(\bar{\mu}, \bar{v})}{\partial(\mu, v)}\right]=\left(c_{n}\left(\mu_{n}^{\prime}, v_{n}^{\prime}\right)\right)^{-2} \rho_{\mu_{n}^{\prime}, v_{n}^{\prime}}^{2 n}\left[n \rho_{\mu_{n}^{\prime}, v_{n}^{\prime}}^{-n-1}\left(\partial_{\mu}\left(\rho_{\mu, v}\right)+\partial_{\nu}\left(\rho_{\mu, v}\right)\right)+1\right]
$$

and then

$$
\left(c_{n}\left(\mu_{n}^{\prime}, v_{n}^{\prime}\right)\right)^{2} \rho_{\mu_{n}^{\prime}, v_{n}^{\prime}}^{-2 n} \operatorname{det}\left[\frac{\partial(\bar{\mu}, \bar{v})}{\partial(\mu, v)}\right] \approx 1 .
$$

This implies $(\mu, v) \rightarrow(\bar{\mu}, \bar{v})$ is invertible in a neighbourhood of $\left(\mu_{n}^{\prime}, v_{n}^{\prime}\right)$.

Now, note that for $\|(\bar{\mu}, \bar{v})\|<r, \max \{\mu, \nu\}<2 \rho_{\mu, \nu}^{-n}$ for $n$ large enough depending on $r$. Hence the domain of the map $(\bar{\mu}, \bar{v}) \rightarrow(\mu, \nu)$ converges to $\mathbb{R}^{2}$, and then $\mu=$ $\mu_{n}(\bar{\mu}, \bar{v})$ and $v=v_{n}(\bar{\mu}, \bar{v})$ is a well defined reparametrization in any large compact set.

Next, we define a map $\psi_{n, \bar{\mu}, \bar{v}}$ from $\mathbb{R}$ to $\mathbb{R}$ by $y=\psi_{n, \bar{\mu}, \bar{v}}(\bar{y})=c_{n} \bar{y}+1$, where $c_{n}=c_{n}$ $(\mu, \nu)$ ( hence $c_{n}$ depends on $\bar{\mu}$ and $\left.\bar{v}\right)$, and we also define $h_{n, \bar{\mu}, \bar{v}}=\left(\psi_{n, \bar{\mu}, \bar{\nu}}\right)^{-1} \circ g_{n, \mu, \nu} \circ$ $\psi_{n, \bar{\mu}, \bar{v}}$. From (4) and (5) and a direct evaluation we obtain

$$
h_{n, \bar{\mu}, \bar{v}}(\bar{y})=\left\{\begin{array}{lc}
-\frac{k_{\mu, \nu}(y)}{k_{0,0}(1)}(-\bar{y})^{\alpha_{\mu, \nu}}+\bar{\mu}, & \frac{a-1}{c_{n}} \leqslant \bar{y}<0, \\
-\frac{\tilde{k}_{\mu, \nu}(y)}{k_{0,0}(1)}(\bar{y})^{\alpha_{\mu, \nu}}+\bar{v}, & 0<\bar{y} \leqslant \frac{b-1}{c_{n}}
\end{array}\right.
$$

and

$$
\partial_{\bar{y}} h_{n, \bar{\mu}, \bar{v}}(\bar{y})=\left\{\begin{array}{lc}
\left(\alpha_{\mu, \nu} \frac{k_{\mu, v}(y)}{k_{0,0}(1)}+\frac{\partial_{y} k_{\mu, v}(y)}{k_{0,0}(1)} c_{n} \bar{y}\right)(-\bar{y})^{\alpha_{\mu, v}-1}, & \frac{a-1}{c_{n}} \leqslant \bar{y}<0, \\
\left(-\alpha_{\mu, \nu} \frac{\tilde{k}_{\mu, v}(y)}{k_{0,0}(1)}-\frac{\partial_{y} \tilde{k}_{\mu, v}(y)}{k_{0,0}(1)} c_{n} \bar{y}\right) \bar{y}^{\alpha_{\mu, v}-1}, & 0<\bar{y} \leqslant \frac{b-1}{c_{n}} .
\end{array}\right.
$$

When $n$ tends to infinite, the parameters $\mu=\rho_{\mu, \nu}^{-n}\left(c_{n} \bar{\mu}+1\right)$ and $v=\rho_{\mu, \nu}^{-n}\left(c_{n} \bar{v}+1\right)$, and $c_{n}$ go to 0 , and thus, from the expresion above we obtain that $h_{n, \bar{\mu}, \bar{v}}$ converges $C^{1}$ uniformly on any compact set in the $\bar{y}$-space to $h_{\bar{\mu}, \bar{v}}$. In a similar way $C^{2}$ convergence on $\bar{y}$ is obtained.

The $(\bar{\mu}, \bar{v})$-convergence in the $C^{0}$ sense is clear from the formulas. For the $C^{1}$ and $C^{2}$ $(\bar{\mu}, \bar{v})$-convergence we need to compute $\partial_{r}\left(\partial_{\bar{y}}^{i} h_{n, \bar{\mu}, \bar{v}}(\bar{y})\right)$ for $i=0,1,2$ and $r=\bar{\mu}, \bar{v}$. We will compute only $\partial_{\bar{\mu}} h_{n, \bar{\mu}, \bar{\nu}}(\bar{y})$. The other ones go on in a similar way and we left them to the reader.

For $\bar{y}<0$, a straightforward computation give us

$$
\begin{aligned}
\partial_{\bar{\mu}} h_{n, \bar{\mu}, \bar{v}}(\bar{y})= & \frac{\partial_{y} k_{\mu, v}(y)}{k_{0,0}(1)}\left[\partial_{\mu} c_{n} \partial_{\bar{\mu}} \mu+\partial_{\nu} c_{n} \partial_{\bar{\mu}} v\right](-\bar{y})^{\alpha_{\mu, \nu}+1} \\
& -\left[\partial_{\mu} k_{\mu, v}\left(c_{n} \bar{y}+1\right) \partial_{\bar{\mu}} \mu+\partial_{\nu} k_{\mu, v}(y) \partial_{\bar{\mu}} v\right](-\bar{y})^{\alpha_{\mu, v}} \\
& -\frac{k_{\mu, \nu}(y)}{k_{0,0}(1)}(-\bar{y})^{\alpha_{\mu, v}}\left[\partial_{\mu} \alpha_{\mu, \nu} \partial_{\bar{\mu}} \mu+\partial_{\nu} \alpha_{\mu, \nu} \partial_{\bar{\mu}} v\right] \log (-\bar{y})+1,
\end{aligned}
$$

and for $\bar{y}>0$ 


$$
\begin{aligned}
\partial_{\bar{\mu}} h_{n, \bar{\mu}, \bar{v}}(\bar{y})= & -\frac{\partial_{y} \tilde{k}_{\mu, \nu}(y)}{k_{0,0}(1)}\left[\partial_{\mu} c_{n} \partial_{\bar{\mu}} \mu+\partial_{\nu} c_{n} \partial_{\bar{\mu}} \nu\right] \bar{y}^{\alpha_{\mu, \nu}+1} \\
& -\left[\partial_{\mu} \tilde{k}_{\mu, \nu}(y) \partial_{\bar{\mu}} \mu+\partial_{\nu} \tilde{k}_{\mu, \nu}(y) \partial_{\bar{\mu}} v\right] \bar{y}^{\alpha_{\mu, \nu}} \\
& -\frac{\tilde{k}_{\mu, \nu}(y)}{k_{0,0}(1)} \bar{y}^{\alpha_{\mu, \nu}}\left[\partial_{\mu} \alpha_{\mu, \nu} \partial_{\bar{\mu}} \mu+\partial_{\nu} \alpha_{\mu, \nu} \partial_{\bar{\mu}} v\right] \log (\bar{y})+1 .
\end{aligned}
$$

We need to estimate $\partial_{\mu} c_{n}, \partial_{\nu} c_{n}, \partial_{\bar{\mu}} \mu$ and $\partial_{\bar{\mu}} \nu$. A direct computation gives:

$$
\partial_{\mu} c_{n}=c_{n}\left[\frac{1}{1-\alpha_{\mu, \nu}} n \rho_{\mu, \nu}^{-1} \partial_{\mu} \rho_{\mu, \nu}+\frac{\partial_{\mu} \alpha_{\mu, \nu}}{\left(1-\alpha_{\mu, \nu}\right)^{2}} \log \left(k_{0,0} \rho_{\mu, \nu}^{n}\right)\right]
$$

and

$$
\partial_{\nu} c_{n}=c_{n}\left[\frac{1}{1-\alpha_{\mu, \nu}} n \rho_{\mu, \nu}^{-1} \partial_{\nu} \rho_{\mu, \nu}+\frac{\partial_{\nu} \alpha_{\mu, \nu}}{\left(1-\alpha_{\mu, \nu}\right)^{2}} \log \left(k_{0,0} \rho_{\mu, \nu}^{n}\right)\right] .
$$

From here we see that $\partial_{\mu} c_{n}=c_{n} U(n)$ and $\partial_{\nu} c_{n}=c_{n} V(n)$, where $U(n)=n p(\mu, v)+$ $q(\mu, v)$ and $V(n)=n \tilde{p}(\mu, v)+\tilde{q}(\mu, v)$ with $p(\mu, v), q(\mu, v), \tilde{p}(\mu, v), \tilde{q}(\mu, v)$ bounded functions. From this and (6) we obtain

$$
\operatorname{det}\left[\frac{\partial(\bar{\mu}, \bar{v})}{\partial(\mu, v)}\right]=\left(\frac{\rho_{\mu, v}^{n}}{c_{n}}\right)^{2}[1+\theta(n)]
$$

where $\theta(n)$ depends on $\mu$ and $v$, and goes to zero when $n$ goes to infinite. From this and the formula

$$
\frac{\partial(\mu, v)}{\partial(\bar{\mu}, \bar{v})}=\left[\frac{\partial(\bar{\mu}, \bar{v})}{\partial(\mu, v)}\right]^{-1}=\left(\operatorname{det}\left[\frac{\partial(\bar{\mu}, \bar{v})}{\partial(\mu, v)}\right]\right)^{-1}\left[\begin{array}{cc}
\partial_{\nu} \bar{v} & -\partial_{\nu} \bar{\mu} \\
-\partial_{\mu} \bar{v} & \partial_{\mu} \bar{\mu}
\end{array}\right]
$$

we obtain

$$
\partial_{\bar{\mu}} \mu=\left(\frac{c_{n}}{\rho_{\mu, \nu}^{n}}\right)^{2} \frac{1}{1+\theta(n)} \partial_{\nu} \bar{v} \quad \text { and } \quad \partial_{\bar{\mu}} v=\left(\frac{c_{n}}{\rho_{\mu, \nu}^{n}}\right)^{2} \frac{-1}{1+\theta(n)} \partial_{\mu} \bar{\nu}
$$

from this expresion and (6) we conclude that

$$
\partial_{\bar{\mu}} \mu \approx \frac{c_{n}}{\rho_{\mu, \nu}^{n}} \quad \text { and } \quad \partial_{\bar{\mu}} v \approx \frac{c_{n}}{\rho_{\mu, \nu}^{n}}
$$

and consequently $\partial_{\bar{\mu}} \mu$ and $\partial_{\bar{\mu}} v$ go to zero when $n$ goes to infinite and the $C^{1}-(\mu, v)$ convergence follows.

To finish the proof of lemma, for $c_{n}^{-1}(a-1) \leqslant \bar{y}<0$, recalling that $y=c_{n} \bar{y}+1$ and using definition for $h_{n, \bar{\mu}, \bar{v}}(\bar{y})$, we have

$$
\begin{aligned}
\partial_{\bar{y}} h_{n, \bar{\mu}, \bar{v}}(\bar{y}) & =\partial_{\bar{y}}\left(\left(\psi_{n, \bar{\mu}, \bar{v}}\right)^{-1} \circ g_{n, \mu, v} \circ \psi_{n, \bar{\mu}, \bar{\nu}}\right)(\bar{y}) \\
& =\rho_{\mu, \nu}^{n} \partial_{y} f_{\mu, \nu}(y) \\
& =\rho_{\mu, \nu}^{n} \frac{\partial_{y} f_{\mu, \nu}(y)}{(1-y)^{\alpha_{\mu, \nu}-1}}(1-y)^{\alpha_{\mu, \nu}-1},
\end{aligned}
$$

but then $\partial_{\bar{y}} h_{n, \bar{\mu}, \bar{v}}(\bar{y})=\frac{1}{k_{0,0}(1)} \frac{\partial_{y} f_{\mu, \nu}(y)}{|1-y|^{\alpha \mu, v}-1}|\bar{y}|^{\alpha_{\mu, \nu}-1}$. 
The same conclusion is obtained for $0<\bar{y}<c_{n}^{-1}(b-1)$. Now, from (2) the first inequality in the lemma follows. The same argument works for the second inequality.

\section{Proof of the main theorem}

In this section we will prove a one dimensional version of Theorem 1 from which Theorem 1 follows.

THEOREM 2.- For each $n \in \mathbb{N}$ large enough there exists a Lebesgue positive measure set $E_{n}$ in the $(\mu, v)$-parameter space such that:

(1) For every $(\mu, v) \in E_{n}$, the critical values $\rho_{\mu, \nu}^{n} \mu$ and $\rho_{\mu, v}^{n} v$ of the function $g_{n, \mu, v}=\left(f_{\mu, \nu}\right)^{n+1}$ have positive Lyapunov exponents, in other words, there exists $\lambda>1$ such that $\left|\left(\left(g_{n, \mu, \nu}\right)^{k}\right)^{\prime}\left(\rho_{\mu, \nu}^{n} \mu\right)\right|>\lambda^{k}$, and $\left|\left(\left(g_{n, \mu, v}\right)^{k}\right)^{\prime}\left(\rho_{\mu, v}^{n} v\right)\right|>\lambda^{k}$ for every $k>0$.

(2) For every $(\mu, v) \in E_{n}$, both critical orbits, i.e. $\left\{\left(\left(g_{n, \mu, \nu}\right)^{k}\right)^{\prime}\left(\rho_{\mu, \nu}^{n} \mu\right)\right\}_{k \in \mathbb{N}}$ and $\left\{\left(\left(g_{n, \mu, v}\right)^{k}\right)^{\prime}\left(\rho_{\mu, \nu}^{n} v\right)\right\}_{k \in \mathbb{N}}$ are dense subsets of the interval $\Theta_{\mu, v}=\left[g_{n, \mu, v}(\eta), \eta\right]$ where $\eta=\max \left\{\rho_{\mu, \nu}^{n} \mu, \rho_{\mu, \nu}^{n} \nu\right\}$.

Remark 2. - Note that our Theorem 1 follows from Theorem 2. In fact, for each $(\mu, \nu) \in E_{n}$, the set $Q_{\mu, \nu}=\bigcap_{T>0} \bigcup_{t>T} X_{\mu, \nu}^{t}\left(\pi^{-1}\left(\Theta_{\mu, \nu}\right)\right)$ is the announced attractor since $\Theta_{\mu, \nu}$ is transitive and the foliation defining $f_{\mu, \nu}$ is contracting.

The proof is quite long and we need to introduce some terminology. For a positive large integer $n$, define the $\mu$-dependent points $p(\mu)$ and $q(\mu)$ by the equations $g_{n, \mu, \mu}(p(\mu))=p(\mu)=g_{n, \mu, \mu}(q(\mu))$.

Note that $a(\mu, \mu)<p(\mu)<1<q(\mu)<b(\mu, \mu)$. Let $\mu_{1}, \mu_{2}$ be defined by

$$
\mu_{1}=\sup \left\{\mu \mid \rho_{\mu, \mu}^{n} \mu \leqslant 1\right\} \quad \text { and } \quad \mu_{2}=\inf \left\{\mu \mid b(\mu, \mu) \leqslant \rho_{\mu, \mu}^{n} \mu\right\} .
$$

The map $w:\left[\mu_{1}, \mu_{2}\right] \rightarrow \mathbb{R}$ defined by $w(\mu)=\rho_{\mu, \mu}^{n} \mu-q(\mu)$ is continuous, $w\left(\mu_{1}\right)<0$ and $w\left(\mu_{2}\right)>0$, hence there is a parameter value $\mu_{n} \in\left[\mu_{1}, \mu_{2}\right]$ such that $\rho_{\mu_{n}, \mu_{n}}^{n} \mu_{n}=$ $q\left(\mu_{n}\right)$, see Fig. 4 .

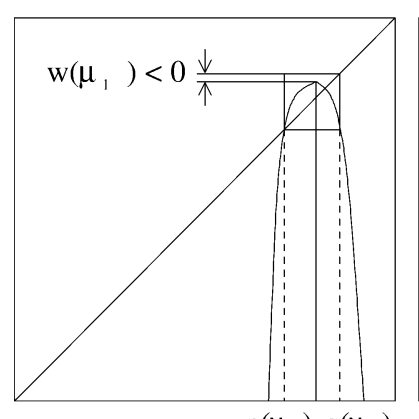

$\mathrm{p}\left(\mu_{1}\right) \mathrm{q}\left(\mu_{1}\right)$
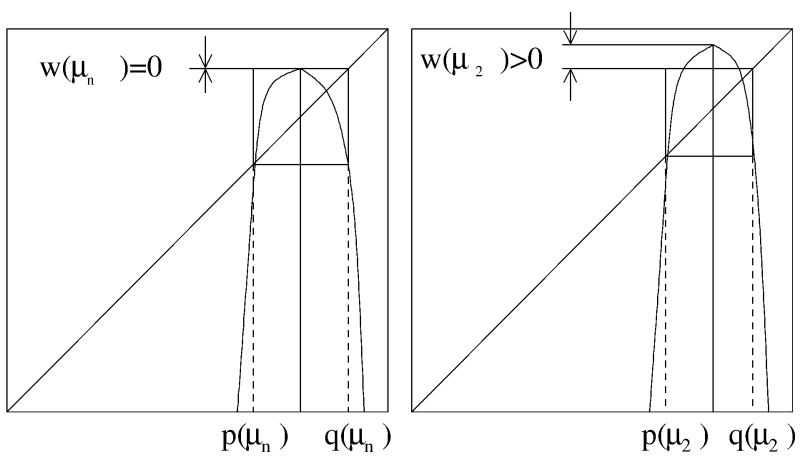

Fig. 4. 
From the definitions of $p_{n}, q_{n}, \mu_{n}$ and $g_{n, \mu_{n}, \mu_{n}}$, we have that for $n$ large enough we get

$$
1-p_{n}=\left(\frac{k_{\mu_{n}, \mu_{n}}\left(p_{n}\right)}{\tilde{k}_{\mu_{n}, \mu_{n}}\left(q_{n}\right)}\right)^{1 / \alpha}\left(q_{n}-1\right)
$$

and

$$
1-p_{n}=\left(\rho_{\mu_{n}, \mu_{n}}^{-n}\left[\left(\frac{k_{\mu_{n}, \mu_{n}}\left(p_{n}\right)}{\tilde{k}_{\mu_{n}, \mu_{n}}\left(q_{n}\right)}\right)^{1 / \alpha}+1\right] \frac{1}{k_{\mu_{n}, \mu_{n}}\left(p_{n}\right)}\right)^{1 /(\alpha-1)}
$$

hence, using (4) $\frac{1-p_{n}}{c_{n}}$ and $\frac{q_{n}-1}{c_{n}}$ are uniformly bounded. This implies that there are parameter values $\bar{\mu}_{n}^{c_{n}}=\bar{v}_{n}$ and points $\bar{q}_{n}=\bar{\mu}_{n}, \bar{p}_{n}$ in the $(\bar{y}, \bar{\mu}, \bar{v})$-space, such that $h_{n, \bar{\mu}_{n}, \bar{v}_{n}}\left(\bar{p}_{n}\right)=\bar{p}_{n}=h_{n, \bar{\mu}_{n}, \bar{v}_{n}}\left(\bar{q}_{n}\right)$.

Furthermore, from the definition of $h_{\bar{\mu}, \bar{v}}$ we can see that there are a unique parameter value $\bar{\mu}_{\infty}=\bar{v}_{\infty}$ and points $\bar{q}=\bar{v}_{\infty}$ and $\bar{p}$ such that $h_{\bar{\mu}_{\infty}, \bar{v}_{\infty}}(\bar{p})=\bar{p}=h_{\bar{\mu}_{\infty}, \bar{v}_{\infty}}(\bar{q})$ and since $h_{n, \bar{\mu}, \bar{\nu}}$ converges uniformly to $h_{\bar{\mu}, \bar{\nu}}$ on any compact set in $\mathbb{R}$ we conclude that $\bar{p}_{n} \rightarrow \bar{p}$, $\bar{q}_{n} \rightarrow \bar{q}$ and $\bar{\mu}_{n}=\bar{v}_{n} \rightarrow \bar{\mu}_{\infty}=\bar{v}_{\infty}$ when $n \rightarrow \infty$.

For each $\theta>0$ consider the straight line $L_{n, \theta}$ in the $(\bar{\mu}, \bar{v})$-plane given by

$$
L_{n, \theta}=\left\{(\bar{\mu}, \bar{v}) \mid \bar{v}=\theta\left(\bar{\mu}-\bar{\mu}_{n}\right)+\bar{v}_{n}\right\} .
$$

Let $m$ and $m_{\theta}$ be the Lebesgue measure in the $(\bar{\mu}, \bar{v})$-plane and in the straight line $L_{n, \theta}$ respectively. Using the Benedick-Carleson techniques, for $n$ large (see [1], [2, Section 2], [10, Section 3], [15]) we will be able to show the existence of a $m_{\theta}$-positive measure subset $\tilde{E}_{n, \theta}$ of $L_{n, \theta}$, having $\left(\bar{\mu}_{n}, \bar{v}_{n}\right)$ as a density point and satisfying the requisites for Theorem 2 in the $(\bar{\mu}, \bar{v})$-parameter setting. Once we have done this, we consider $\tilde{E}_{n}=\bigcup_{\theta} \tilde{E}_{n, \theta}$. Fubini's theorem implies $m\left(\tilde{E}_{n}\right)>0$. Now, since the reparametrization $(\mu, v) \rightarrow(\bar{\mu}, \bar{v})$ is a diffeomorphism, we get a set $E_{n}$ in the $(\mu, v)$ plane satisfying the requirements of Theorem 2 because $\psi_{n, \bar{\mu}, \bar{\nu}}$ is an affine map.

Before begining the set up of the $\tilde{E}_{n, \theta}$, following the steps on [15] we will prove that the maximal orbits outside of a neighbourhood of the critical point, having length bigger than $K_{0}$ (some $K_{0}$ ) have exponential growth. This fact is established in Proposition 2 and it is a key step in order to apply Benedick-Carleson techniques. In Lemma 5 we will show that under the basic assumption (BA) the increasing in the derivative after a binding period (see definitions before Lemma 5) fully compensates the small factor introduced in the derivative by the orbit point of the critical value passing close to the critical point. As a consequence, we can establish via an inductive argument conditions (condition (FA), given before Proposition 3) to guarantee the exponential growing of the derivatives at the critical values. This is stated in Proposition 3.

To prove that the maximal orbits outside of a neighbourhood of the critical point have exponential growth we follow [15]. The nonsymmetry of the invariant interval is the main difference here.

LEMMA 3. - There exists a constant $C>0$ such that, for every small $\delta_{0}$ there are constants $\lambda_{1}=\lambda_{1}\left(\delta_{0}\right)>1, N_{1}=N_{1}\left(\delta_{0}\right), K_{0}=K_{0}\left(\delta_{0}\right)$ and $a_{1}=a_{1}\left(\delta_{0}\right)$ satisfying the following property: For every $n>N_{1}$, for every $(\bar{\mu}, \bar{v})$ such that $\left|(\bar{\mu}, \bar{v})-\left(\bar{\mu}_{n}, \bar{v}_{n}\right)\right|<a_{1}$, and $\bar{y}$ such that $\left|h_{n, \bar{\mu}, \bar{v}}^{i}(\bar{y})\right| \geqslant \delta_{0}, i=0, \ldots, k-1$, but $\left|h_{n, \bar{\mu}, \bar{v}}^{k}(\bar{y})\right|<\delta_{0}$, then 


$$
\left|\left(h_{n, \bar{\mu}, \bar{\nu}}^{k}\right)^{\prime}(\bar{y})\right|> \begin{cases}C & \text { if } k<K_{0}, \\ \lambda_{1}^{k} & \text { if } k \geqslant K_{0} .\end{cases}
$$

Proof. - Given $\varepsilon>0$ small, $0<\delta_{0}<\frac{1}{2} \min \{|p|, q\}$ and $k \in \mathbb{N}$, let $W=[p-\varepsilon, q+$ $\varepsilon] \backslash\left(-\delta_{0}, \delta_{0}\right), U_{k}(\bar{\mu}, \bar{v})=\left\{\bar{y} \in W \mid h_{\bar{\mu}, \bar{v}}^{i}(\overline{\bar{y}}) \in W, i=0, \ldots, k\right\}$ and $U_{k}(n, \bar{\mu}, \bar{v})=\{\bar{y} \in$ $\left.W / h_{n, \bar{\mu}, \bar{v}}^{i}(\bar{y}) \in W, i=0, \ldots, k\right\}$.

The set $\Delta=\bigcap_{k \geqslant 0} U_{k}\left(\bar{\mu}_{\infty}, \bar{v}_{\infty}\right)$ is compact, $h_{\bar{\mu}_{\infty}, \bar{v}_{\infty}}$-invariant, without sinks (Singer's Theorem), its periodic points are hyperbolic (Minimum Principle) and away from the critical point. From Mañé's Theorem [8] (see also Misiurewicz's Theorem in the same reference), we have that $\Delta$ is hyperbolic. This means that there are constants $\tilde{C}>0$ and $\lambda>1$ such that for every $\bar{y} \in \Delta$ and $k \in \mathbb{N}$ we have $\left|\left(h_{\bar{\mu}_{\infty}, \bar{v}_{\infty}}^{k}\right)^{\prime}(\bar{y})\right| \geqslant \tilde{C} \lambda^{k}$. From this we can conclude that there are constants $\lambda_{1}>1, k_{0}, N_{1}$ and $a_{1}$ depending on $\delta_{0}$, and an open neighbourhood $V$ of $\Delta$ such that for every $k \geqslant k_{0}, n \geqslant N_{1}$ and $(\bar{\mu}, \bar{v})$ satisfying $\left|(\bar{\mu}, \bar{v})-\left(\bar{\mu}_{n}, \bar{v}_{n}\right)\right|<a_{1}$ then

$$
\forall \bar{y}, h_{n, \bar{\mu}, \bar{v}}^{i}(\bar{y}) \in V, 0 \leqslant i<k-1 \Rightarrow\left|\left(h_{n, \bar{\mu}, \bar{v}}^{k}\right)^{\prime}(\bar{y})\right| \geqslant \lambda_{1}^{k} .
$$

Note that we can take $k_{0}=\min \left\{k \mid \tilde{C} \lambda^{k}>1\right\}$ and $1<\lambda_{1}<\lambda(\tilde{C})^{1 / k_{0}}$.

Furthermore, since $U_{k+1}\left(\bar{\mu}_{\infty}, \bar{v}_{\infty}\right) \subset U_{k}\left(\bar{\mu}_{\infty}, \bar{v}_{\infty}\right)$ for $a_{1}$ small enough and $N_{1}$ large, there is a $k_{1}$ (depending only on $\delta_{0}$ ) such that $U_{k_{1}}(n, \bar{\mu}, \bar{v}) \subset V \cap W$. Let $\bar{y} \in[p-$ $\varepsilon, q+\varepsilon] \backslash V$ (and hence $\bar{y} \notin U_{k_{1}}(n, \bar{\mu}, \bar{v})$ ), then there exists a time $j<k_{1}$ such that $\left|h_{n, \bar{\mu}, \bar{v}}^{j}(\bar{y})\right|<\delta_{0}$. Let consider $K_{0}=k_{0}+k_{1}$ and $C=\frac{1}{2} \min \left\{\frac{|p|}{q}, \frac{q}{|p|}\right\}$. We have the following claim: If $\bar{y}$ is such that for some $i \leqslant K_{0}$ and $\left|h_{n, \bar{\mu}, \bar{v}}^{i}(\bar{y})\right|<\delta_{0}$ then

$$
\left|\left(h_{n, \bar{\mu}, \bar{\nu}}^{i}\right)^{\prime}(\bar{y})\right| \geqslant C .
$$

In fact, the Minimum Principle applied to $h=h_{\bar{\mu}_{\infty}, \bar{v}_{\infty}}$ gives that $\left|\left(h^{i}\right)^{\prime}(\bar{y})\right|>\frac{3}{2} C$, indeed, suppose that for some $\bar{y},\left|h^{i}(\bar{y})\right|<\delta_{0}$ and $\left|\left(h^{i}\right)^{\prime}(\bar{y})\right|<\frac{3}{2} C$. Let $\left[\bar{y}_{1}, \bar{y}_{2}\right]$ be the small interval containing $\bar{y}$ such that $\bar{y}_{1}$ and $\bar{y}_{2}$ are local extremes of $h^{i}$. From the choice of $C$ and $\delta_{0}$ we have that the map $\bar{y} \rightarrow\left|\left(h^{i}\right)^{\prime}(\bar{y})\right|$ has a minimun in $\left(\bar{y}_{1}, \bar{y}_{2}\right)$ but, by the Minimun Principle this is not possible. Taking $a_{1}$ small enough and $N_{1}$ large the claim follows.

Now, let $\bar{y}$ be as in the statement of the lemma, then for a suitable choice of $m$, indeed $m=\min \left\{\{k\} \cup\left\{i / h_{n, \bar{\mu}, \bar{v}}^{i}(\bar{y}) \notin V\right\}\right\}$ we have that $h_{n, \bar{\mu}, \bar{v}}^{m}(\bar{y}) \notin U_{k_{1}}(n, \bar{\mu}, \bar{v})$ and then $j=k-m<k_{1}$. Applying the chain rule, (7) and (8) we obtain

$$
\begin{aligned}
\left|\left(h_{n, \bar{\mu}, \bar{\nu}}^{k}\right)^{\prime}(\bar{y})\right| & \geqslant \begin{cases}C & \text { if } m<k_{0}, \\
\left|\left(h_{n, \bar{\mu}, \bar{\nu}}^{j}\right)_{\left.\left(h_{n, \bar{\mu}, \bar{v}}^{m} \bar{y}\right)\right)}^{\prime}\right|\left|\left(h_{n, \bar{\mu}, \bar{v}}^{m}\right)^{\prime}(\bar{y})\right| & \text { if } m \geqslant k_{0},\end{cases} \\
& \geqslant \begin{cases}C & \text { if } m<k_{0}, \\
C \lambda_{1}^{m} & \text { if } m \geqslant k_{0} .\end{cases}
\end{aligned}
$$

Thus, for $k_{0}$ large enough we obtain (by decreasing $\lambda_{1}$ ), a new constant $\lambda_{1}>1$ such that

$$
\left|\left(h_{n, \bar{\mu}, \bar{v}}^{k}\right)^{\prime}(\bar{y})\right| \geqslant \begin{cases}C & \text { if } k<K_{0} \\ \lambda_{1}^{k} & \text { if } k \geqslant K_{0}\end{cases}
$$


LEMMA 4. - Given $\delta_{0}$ small enough and $C>0$ there exists $\lambda_{2}>1$, not depending on $\delta_{0}$, with the following property: for $\delta<\delta_{0}$ there are $N_{2}=N_{2}(\delta) \in \mathbb{N}$ and $a_{2}=a_{2}(\delta)>0$ such that for every $n>N_{2}$, for any $(\bar{\mu}, \bar{v})$ with $\left|(\bar{\mu}, \bar{v})-\left(\bar{\mu}_{n}, \bar{v}_{n}\right)\right|<a_{2}$, and $\bar{y}$, $\delta \leqslant|\bar{y}|<\delta_{0}$, there is $l=l\left(a_{2}, \bar{y}, \delta_{0}\right)$ such that $\left|h_{n, \bar{\mu}, \bar{v}}^{j}(\bar{y})\right|>\delta_{0}$ for $j=1, \ldots, l$ and $\left|\left(h_{n, \bar{\mu}, \bar{\nu}}^{l}\right)^{\prime}(\bar{y})\right|>\lambda_{2}^{l}$. Furthermore, there are constants $L=L\left(\delta_{0}\right)$ and $M=M\left(\delta, \delta_{0}\right)$ such that $L<l<M$ and besides $C \lambda_{2}^{L}>1$.

Proof. - Let define $M_{\varepsilon}=M_{\varepsilon}(n, \bar{\mu}, \bar{v})=\max \left\{\left|\left(h_{n, \bar{\mu}, \bar{v}}\right)^{\prime}(x)\right|: p_{n}-\varepsilon \leqslant x \leqslant p_{n}+\varepsilon\right\}$ and $m_{\varepsilon}=m_{\varepsilon}(n, \bar{\mu}, \bar{v})=\min \left\{\left|\left(h_{n, \bar{\mu}, \bar{v}}\right)^{\prime}(x)\right|: p_{n}-\varepsilon \leqslant x \leqslant p_{n}+\varepsilon\right\}$. When $n$ tends to infinite, $h_{n, \bar{\mu}, \bar{v}}$ converges uniformly on compact sets to $h_{\bar{\mu}, \bar{v}}$ and thus, given $\zeta>0$, there are constants $\tau>0, \bar{\varepsilon}>0$ and $N_{2} \in \mathbb{N}$ such that $\left|(\bar{\mu}, \bar{v})-\left(\bar{\mu}_{\infty}, \bar{v}_{\infty}\right)\right|<\tau, \varepsilon<\bar{\varepsilon}, n \geqslant N_{2} \Rightarrow$ $1-\zeta<m_{\varepsilon} / M_{\varepsilon}<1$.

From this, and since $\alpha=\alpha_{\mu, \nu} \approx \alpha_{0,0}$, we can choose constants $a_{2}>0, \varepsilon>0, N_{2} \in \mathbb{N}$ and $\lambda>1$, such that $n \geqslant N_{2},\left|(\bar{\mu}, \bar{v})-\left(\bar{\mu}_{n}, \bar{v}_{n}\right)\right|<a_{2} \Rightarrow m_{\varepsilon} M_{\varepsilon}^{(1-\alpha) / \alpha}>\lambda>1$. Note that $\lambda$ is independent of $\delta_{0}$. Next, let $\delta_{0}$ be a small positive constant and fix $\delta, 0<\delta<\delta_{0}$. Write $h=h_{n, \bar{\mu}, \bar{v}}$. Given $\bar{y}, \delta \leqslant \bar{y} \leqslant \delta_{0}$, let $l$ be the first positive integer such that $h^{l}\left(h^{2}(\bar{y})\right)>p_{n}+\varepsilon$. Notice that from the uniform convergence of $h_{n, \bar{\mu}_{n}, \bar{\nu}_{n}}$ to $h_{\bar{\mu}_{\infty}, \bar{v}_{\infty}}$, there are constants $N_{2} \in \mathbb{N}, L=L\left(\delta_{0}\right)$ and $M=M\left(\delta, \delta_{0}\right)$ such that for $n \geqslant N_{2}$ we have $L<l<M$. We also observe that for $N_{2}$ large and $a_{2}$ small enough we can get $\left|h\left(0^{ \pm}\right)-q_{n}\right|+\left|h^{l}\left(h\left(q_{n}\right)\right)-p_{n}\right|$ as small as we want because $h_{n, \bar{\mu}_{n}, \bar{\nu}_{n}}\left(q_{n}\right)=p_{n}$, where $h\left(0^{ \pm}\right)=\lim _{\bar{y} \rightarrow 0^{ \pm}} h(\bar{y})$.

Now, using the above comments, the chain rule, the mean value theorem and constants $K_{1}$ and $K_{2}$ given by inequality (3) in the renormalization lemma, we obtain the following estimates:

$$
\begin{aligned}
\varepsilon & <h^{l}\left(h^{2}(\bar{y})\right)-p_{n} \\
& \leqslant\left|h^{l}\left(h^{2}(\bar{y})\right)-h^{l}\left(h^{2}\left(0^{ \pm}\right)\right)\right|+\left|h^{l}\left(h^{2}\left(0^{ \pm}\right)\right)-h^{l}\left(h\left(q_{n}\right)\right)\right|+\left|h^{l}\left(h\left(q_{n}\right)\right)-p_{n}\right| \\
& \leqslant M_{\varepsilon}^{l} C_{2} K_{2}|\bar{y}|^{\alpha}+M_{\varepsilon}^{l} C_{2}\left|h\left(0^{ \pm}\right)-q_{n}\right|+\left|h^{l}\left(h\left(q_{n}\right)\right)-p_{n}\right|,
\end{aligned}
$$

where $C_{2}$ is a positive constant close to $\left|\left(h_{\bar{\mu}_{\infty}, \bar{v}_{\infty}}\right)^{\prime}(q)\right|$. Solving this inequality for $|\bar{y}|$ we obtain

$$
|\bar{y}|>\left\{\frac{1}{M_{\varepsilon}^{l} C_{2} K_{2}}\left[\varepsilon-M_{\varepsilon}^{l} C_{2}\left|h\left(0^{ \pm}\right)-q_{n}\right|-\left|h^{l}\left(h\left(q_{n}\right)\right)-p_{n}\right|\right]\right\}^{1 / \alpha} .
$$

Furthermore, for a positive constant $C_{1}$ close to $\left|\left(h_{\bar{\mu}_{\infty}, \bar{v}_{\infty}}\right)^{\prime}(q)\right|$ we also have $\left|\left(h^{l+2}\right)^{\prime}(\bar{y})\right| \geqslant$ $m_{\varepsilon}^{l} C_{1} K_{1}|\bar{y}|^{\alpha-1}$ and then,

$$
\left|\left(h^{l+2}\right)^{\prime}(\bar{y})\right| \geqslant m_{\varepsilon}^{l} C_{1} K_{1} K_{2}^{\frac{1-\alpha}{\alpha}}\left\{\frac{\varepsilon}{M_{\varepsilon}^{l} C_{2}}-\left|h\left(0^{ \pm}\right)-q_{n}\right|-\frac{\left|h^{l}\left(h\left(q_{n}\right)\right)-p_{n}\right|}{M_{\varepsilon}^{l} C_{2} K_{2}}\right\}^{\frac{\alpha-1}{\alpha}} .
$$

Now, if we consider $\bar{\mu}=\bar{\mu}_{n}, \bar{v}=\bar{v}_{n}$ and $L$ large (this last condition occurs with $\delta_{0}$ small), then for some $\lambda_{1}>1$ the above inequality and the choice of $\varepsilon$ leads us to

$$
\begin{aligned}
\left|\left(h^{l+2}\right)^{\prime}(\bar{y})\right| & \geqslant\left(m_{\varepsilon} M_{\varepsilon}^{\frac{1-\alpha}{\alpha}}\right)^{l} \varepsilon^{\frac{\alpha-1}{\alpha}} K_{2}^{\frac{1-\alpha}{\alpha}} C_{1} C_{2}^{\frac{1-\alpha}{\alpha}} K_{1} \\
& \geqslant \bar{C} \lambda^{l}, \quad \text { with } \bar{C}=\varepsilon^{\frac{\alpha-1}{\alpha}} K_{2}^{\frac{1-\alpha}{\alpha}} C_{1} C_{2}^{\frac{1-\alpha}{\alpha}} K_{1} \\
& \geqslant \lambda_{1}^{l+2} .
\end{aligned}
$$


Since $L<l<M$, taking $a_{2}$ small enough, we can find $\lambda_{2}, 1<\lambda_{2}<\lambda_{1}$, independent of $\delta_{0}$, such that for $(\bar{\mu}, \bar{v})$ with $\left|(\bar{\mu}, \bar{v})-\left(\bar{\mu}_{n}, \bar{v}_{n}\right)\right|<a_{2}$ then $\left|\left(h^{l+2}\right)^{\prime}(\bar{y})\right| \geqslant \lambda_{2}^{l+2}$. Finally, given any positive constant $C$ we can choose $\delta_{0}$ small enough to obtain $C \lambda_{2}^{L}>1$. This ends the proof of the lemma.

Proposition 2. - There exist $K_{0} \in \mathbb{N}, C>0$ and $\lambda_{0}>1$ satisfying the following property: For every $\delta>0$ small enough, there exist $a_{0}=a_{0}(\delta)$ and $N_{0}=N_{0}(\delta)$ such that for every $n>N_{0},(\bar{\mu}, \bar{v})$ with $\left|(\bar{\mu}, \bar{v})-\left(\bar{\mu}_{n}, \bar{v}_{n}\right)\right|<a_{0}$, and $\bar{y}$ such that $\left|h_{n, \bar{\mu}, \bar{v}}^{i}(\bar{y})\right| \geqslant \delta$ for $i=0, \ldots, k-1$ but $\left|h_{n, \bar{\mu}, \bar{v}}^{k}(\bar{y})\right|<\delta$, we have

$$
\left|\left(h_{n, \bar{\mu}, \bar{\nu}}^{k}\right)^{\prime}(\bar{y})\right| \geqslant \begin{cases}C & \text { if } k<K_{0}, \\ \lambda_{0}^{k} & \text { if } k \geqslant K_{0} .\end{cases}
$$

Proof.-Take $C>0$ as in Lemma 3 and take $\delta_{0}>0$ small enough so that Lemma 4 is true for the pair $\left(C, \delta_{0}\right)$. Let $\lambda_{1}=\lambda_{1}\left(\delta_{0}\right), a_{1}\left(\delta_{0}\right), N_{1}\left(\delta_{0}\right), K_{0}=K_{0}\left(\delta_{0}\right)$ and $L=L\left(\delta_{0}\right)$ as in Lemma 3 applied to the pair $\left(C, \delta_{0}\right)$. Given $\delta<\delta_{0}$, let $\lambda_{2}$, $a_{2}(\delta)$ and $N_{2}(\delta)$ be determined by Lemma 4 applied to the pair $\left(C, \delta_{0}\right)$. Define $a_{0}=\min \left\{a_{1}\left(\delta_{0}\right), a_{2}(\delta)\right\}$ and $N_{0}=\max \left\{N_{1}\left(\delta_{0}\right), N_{2}(\delta)\right\}$. Let $n>N_{0}$ and $(\bar{\mu}, \bar{v})$ with $\left|(\bar{\mu}, \bar{v})-\left(\bar{\mu}_{n}, \bar{v}_{n}\right)\right|<a_{0}$. Write $h=h_{n, \bar{\mu}, \bar{v}}$, and let $\bar{y}$ be as in the statement of the proposition. We consider two possibilities: (i) $\delta \leqslant|\bar{y}|<\delta_{0}$ and (ii) $\delta_{0} \leqslant|\bar{y}|$. In the first one we decompose the orbit block $\left\{h^{i}(\bar{y})\right\}_{i=0}^{i=k}$ in blocks $\left\{\bar{y}=y_{0}, h\left(y_{0}\right), \ldots, h^{l_{0}}\left(y_{0}\right)=\right.$ $\left.x_{0}\right\},\left\{h\left(x_{0}\right), \ldots, h^{k_{0}}\left(x_{0}\right)=y_{1}\right\},\left\{h\left(y_{1}\right), \ldots, h^{l_{1}}\left(y_{1}\right)=x_{1}\right\},\left\{h\left(x_{1}\right), \ldots, h^{k_{1}}\left(x_{1}\right)=y_{2}\right\}$, $\left\{h\left(y_{2}\right), \ldots, h^{l_{2}}\left(y_{2}\right)=x_{2}\right\},\left\{h\left(x_{2}\right), \ldots, h^{k_{2}}\left(x_{2}\right)=y_{3}\right\}, \ldots,\left\{h\left(y_{w}\right), \ldots, h^{l_{w}}\left(y_{w}\right)=x_{w}\right\}$, $\left\{h\left(x_{w}\right), \ldots, h^{k_{w}}\left(x_{w}\right)=h^{k}(\bar{y})\right\}$, where $l_{i} \geqslant L$ and $k_{i}$ are inductively defined by applying Lemmas 4 and 3 to $y_{i}$ and $x_{i}$ respectively. Let $k_{i_{1}}, \ldots, k_{i_{r}}$ be the integers satisfying $k_{i j} \geqslant K_{0}$, and rewrite those integers $k_{i}$ such that $k_{i}<K_{0}$ as $s_{0}, s_{1}, \ldots, s_{u-1}$ preserving the appearance order $l_{0} \prec k_{0} \prec l_{1} \prec k_{1} \prec \cdots \prec l_{w} \prec k_{w}$, that is, $s_{0}$ is the first integer $k_{i}$ such that $k_{i}<K_{0}, s_{1}$ is the second one and so on. It is clear that $u \leqslant w, s_{j}<K_{0}$ and $k=\left(l_{0}+s_{0}\right)+\cdots+\left(l_{u-1}+s_{u-1}\right)+l_{u}+\cdots+l_{w}+k_{i_{1}}+\cdots+k_{i_{r}}$. Applying the chain rule, Lemmas 3 and 4 and after some rearrangement, we obtain

$$
\left|\left(h^{k}\right)^{\prime}(\bar{y})\right| \geqslant\left(\lambda_{2}^{l_{0}} C\right) \cdots\left(\lambda_{2}^{l_{u-1}} C\right) \lambda_{2}^{l_{u}+\cdots+l_{w}} \lambda_{1}^{k_{i_{1}}+\cdots+k_{i_{r}}} .
$$

From Lemma 4 we have that if $l>L$ then $C \lambda_{2}^{L}>1$, and we obtain $\lim _{\lambda \rightarrow 1^{+}} l \log \left(\lambda_{2} / \lambda\right)=$ $l \log \lambda_{2}>-\log C=\lim _{\lambda \rightarrow 1^{+}} \log \left(\lambda^{K_{0}} / C\right)$, and consequently we can take $\lambda_{0}, 1<\lambda_{0}<$ $\min \left\{\lambda_{1}, \lambda_{2}\right\}$ such that if $l>L$ then $C \lambda_{2}^{l}>\lambda_{0}^{l+K_{0}}$. In particular, for $l_{i}>L$ and since $s_{i}<K_{0}, i=0, \ldots, u-1$, we have $C \lambda_{2}^{l_{i}}>\lambda_{0}^{l_{i}+s_{i}}$, and then $\left|\left(h^{k}\right)^{\prime}(\bar{y})\right| \geqslant \lambda_{0}^{k}$.

Note that if $k<K_{0}$ then $k_{1}=k$ and in this case we have $\left|\left(h^{k}\right)^{\prime}(\bar{y})\right| \geqslant C$.

Finally, if $\delta_{0} \leqslant|\bar{y}|$, the same arguments as in the $\delta \leqslant|\bar{y}| \leqslant \delta_{0}$ case work, the only difference here is that we do not have the first block $\left\{\bar{y}=y_{0}, h\left(y_{0}\right), \ldots, h^{l_{0}}\left(y_{0}\right)=x_{0}\right\}$, and the second one must be changed by $\left\{\bar{y}=x_{0}, h\left(x_{0}\right), \ldots, h^{k_{0}}\left(x_{0}\right)=y_{1}\right\}$. This ends the proof of proposition.

Given $(\bar{\mu}, \bar{v}) \in L_{n, \theta}=\left\{(\bar{\mu}, \bar{v}) / \bar{v}=\theta\left(\bar{\mu}-\bar{\mu}_{n}\right)+\bar{\mu}\right\}$ set $h_{\bar{\mu}}=h_{n, \bar{\mu}, \bar{v}}$. For a small $\gamma>0$, let define $\tilde{E}_{n, \theta}(\gamma) \subset L_{n, \theta}$ as the set of parameters $(\bar{\mu}, \bar{v})$ satisfying the following basic assumption:

$$
\left|h_{\bar{\mu}}^{j}(\bar{\mu})\right| \geqslant \mathrm{e}^{-\gamma j} \quad \text { and } \quad\left|h_{\bar{\mu}}^{j}(\bar{v})\right| \geqslant \mathrm{e}^{-\gamma j} \quad \forall j \geqslant 1 .
$$


Let $\beta>0,(\bar{\mu}, \bar{v}) \in \tilde{E}_{n, \theta}(\gamma)$, and $\delta>0$ small. For a positive integer $k$ such that $\left|h_{\bar{\mu}}^{k}(\bar{\mu})\right| \leqslant \delta$, define the binding period associated to the parameter $(\bar{\mu}, \bar{v})$ for the return $h_{\bar{\mu}}^{k}(\bar{\mu})$ of the critical value $\bar{\mu}$ as the maximal interval $[k+1, k+s]$ such that for $1 \leqslant j \leqslant s$

$$
\left|h_{\bar{\mu}}^{k+j}(\bar{\mu})-h_{\bar{\mu}}^{j-1}(\bar{\mu})\right|<\mathrm{e}^{-\beta j}, \quad \text { if } h_{\bar{\mu}}^{k}(\bar{\mu})<0,
$$

or

$$
\left|h_{\bar{\mu}}^{k+j}(\bar{\mu})-h_{\bar{\mu}}^{j-1}(\bar{v})\right|<\mathrm{e}^{-\beta j}, \quad \text { if } h_{\bar{\mu}}^{k}(\bar{\mu})>0
$$

holds.

Thus, during the binding period, the orbit of $h_{\bar{\mu}}^{k+1}(\bar{\mu})$ is close to that of $\bar{\mu}$ or $\bar{v}$ depending on above conditions. In the same way, we define the binding period associated to the parameter $(\bar{\mu}, \bar{v})$ for the return $h_{\bar{\mu}}^{k}(\bar{v})$ of the critical value $\bar{v}$.

LEMMA 5. - For a convenient choosing of $\gamma, \beta$ and $\delta$, there are positive constants $\widetilde{K}, D, A, k_{1}$ and $\tau$ depending only on $\gamma$ and $\beta$, such that for both critical values $\bar{\eta}=\bar{\mu}$ or $\bar{\eta}=\bar{v}$ of $h_{\bar{\mu}}=h_{n, \bar{\mu}, \bar{v}}$, with $(\bar{\mu}, \bar{v}) \in \tilde{E}_{n, \theta}(\gamma)$, we have: If $h_{\bar{\mu}}^{k}(\bar{\eta}) \in\left(\mathrm{e}^{-\gamma k}, \delta\right)$ for some $k>k_{1}$ and $\left|\left(h_{\bar{\mu}}^{j}\right)^{\prime}(\bar{\eta})\right|>\lambda_{1}^{j}, j=1, \ldots, k-1$ for some $\lambda_{1}, 1<\lambda_{1}<\lambda_{0}\left(\lambda_{0}\right.$ as in Proposition 2), then

(1)

$$
\frac{1}{A}<\frac{\left|\left(h_{\bar{\mu}}^{j}\right)^{\prime}(x)\right|}{\left|\left(h_{\bar{\mu}}^{j}\right)^{\prime}(y)\right|}<A \quad \text { for all } x, y \in\left[h_{\bar{\mu}}^{k+1}(\bar{\eta}), \bar{v}\right] \text { and } 1 \leqslant j \leqslant s,
$$

where $s$ is the binding period associated to the parameter $(\bar{\mu}, \bar{v})$ for the return $h_{\bar{\mu}}^{k}(\bar{\eta})$ of the critical value $\bar{\eta}$ of $h_{\bar{\mu}}$.

(2)

$$
\frac{r}{\beta+\log D}-1 \leqslant s \leqslant \frac{r \alpha_{\mu, \nu}-\log \left(\widetilde{K} A^{-1}\right)}{\beta+\log \lambda_{1}}, \quad \text { where } \mathrm{e}^{-r}=\left|h_{\bar{\mu}}^{k}(\bar{\eta})\right| .
$$

$$
\left|\left(h_{\bar{\mu}}^{s+1}\right)^{\prime}\left(h_{\bar{\mu}}^{k}(\bar{\eta})\right)\right| \geqslant \tau \exp \left[\left(\frac{\log \lambda_{1}}{\alpha_{\mu, v}}-\frac{\alpha_{\mu, v}-1}{\alpha_{\mu, v}} \beta\right)(s+1)\right]>1 .
$$

Similar results can be obtained if $h_{\bar{\mu}}^{k}(\bar{\eta}) \in\left(-\delta,-\mathrm{e}^{-k \gamma}\right)$, but in this case we have to change $\left[h_{\bar{\mu}}^{k+1}(\bar{\eta}), \bar{v}\right]$ by $\left[h_{\bar{\mu}}^{k+1}(\bar{\eta}), \bar{\mu}\right]$ in conclusion (1) above.

Proof. - First, we observe that taking $\gamma<\beta$ then $\mathrm{e}^{-(j+1) \beta}<\mathrm{e}^{-j \gamma}$. Since $\left|h_{\bar{\mu}}^{j}(\bar{\mu})\right|>$ $\mathrm{e}^{-j \gamma}$ and $s$ is the binding period associated to $(\bar{\mu}, \bar{v})$ for the return $h_{\bar{\mu}}^{k}(\bar{\eta})$ of the critical value $\bar{\eta}$ of $h_{\bar{\mu}}$, we conclude that $0 \notin\left(h_{\bar{\mu}}^{k+1+j}(\bar{\eta}), h_{\bar{\mu}}^{j}(\bar{v})\right)$ for $j<s$. Consequently, if $x \in$ $\left(h_{\bar{\mu}}^{k+1}(\bar{\eta}), \bar{v}\right)$ then $0 \notin\left(h_{\bar{\mu}}^{j}(x), h_{\bar{\mu}}^{j}(\bar{v})\right) \subset\left(h_{\bar{\mu}}^{k+1+j}(\bar{\eta}), h_{\bar{\mu}}^{j}(\bar{v})\right)$. From the second inequality in (3), for all $j \leqslant s-1$ we can find $\xi_{j} \in\left(h_{\bar{\mu}}^{j}(x), h_{\bar{\mu}}^{j}(\bar{v})\right)$ such that

$$
\begin{aligned}
\left|h_{\bar{\mu}}^{\prime}\left(h_{\bar{\mu}}^{j}(x)\right)-h_{\bar{\mu}}^{\prime}\left(h_{\bar{\mu}}^{j}(\bar{v})\right)\right| & \leqslant K_{2}|\xi|^{\alpha_{\mu, v}-2}\left|h_{\bar{\mu}}^{j}(x)-h_{\bar{\mu}}^{j}(\bar{v})\right| \\
& \leqslant K_{2}|\xi|^{\alpha_{\mu, \nu}-2}\left|h^{k+1+j}(\bar{\eta})-h^{j}(\bar{v})\right|,
\end{aligned}
$$


and from here

$$
\begin{aligned}
\left|h_{\bar{\mu}}^{\prime}\left(h_{\bar{\mu}}^{j}(x)\right)-h_{\bar{\mu}}^{\prime}\left(h_{\bar{\mu}}^{j}(\bar{v})\right)\right| & \leqslant K_{2} \mathrm{e}^{-\gamma\left(\alpha_{\mu, \nu}-2\right) j}\left|h^{k+1+j}(\bar{\eta})-h^{j}(\bar{v})\right| \\
& \leqslant K_{2} \mathrm{e}^{-\gamma\left(\alpha_{\mu, v}-2\right) j-(j+1) \beta} .
\end{aligned}
$$

Furthermore, $\left|h_{\bar{\mu}}^{\prime}\left(h_{\bar{\mu}}^{j}(\bar{\eta})\right)\right| \geqslant K_{1}\left|h_{\bar{\mu}}^{j}(\bar{\eta})\right|^{\alpha_{\mu, \nu}-1} \geqslant K_{1} \mathrm{e}^{\left.-j \gamma^{\left(\alpha_{\mu, \nu}-1\right.}\right)}$.

Now,

$$
\begin{aligned}
\left|\frac{\left(h_{\bar{\mu}}^{j}\right)^{\prime}(x)}{\left(h_{\bar{\mu}}^{j}\right)^{\prime}(\bar{\eta})}\right| & =\prod_{i=0}^{j-1}\left[1+\frac{\left|h_{\bar{\mu}}^{\prime}\left(h_{\bar{\mu}}^{i}(x)\right)\right|-\left|h_{\bar{\mu}}^{\prime}\left(h_{\bar{\mu}}^{i}(\bar{\eta})\right)\right|}{\left|h_{\bar{\mu}}^{\prime}\left(h_{\bar{\mu}}^{i}(\bar{\eta})\right)\right|}\right] \\
& \leqslant \exp \left[\sum_{i=0}^{j-1} \frac{\left|h_{\bar{\mu}}^{\prime}\left(h_{\bar{\mu}}^{i}(x)\right)-h_{\bar{\mu}}^{\prime}\left(h_{\bar{\mu}}^{i}(\bar{\eta})\right)\right|}{\left|h_{\bar{\mu}}^{\prime}\left(h_{\bar{\mu}}^{i}(\bar{\eta})\right)\right|}\right] \\
& \leqslant \exp \left[\sum_{i=0}^{j-1} \frac{K_{2} \mathrm{e}^{-\gamma\left(\alpha_{\mu, v}-2\right) j-(j+1) \beta}}{K_{1} \mathrm{e}^{-i \gamma\left(\alpha_{\mu, v}-1\right)}}\right] \\
& <\exp \left[\frac{K_{2}}{K_{1}} \mathrm{e}^{-\beta} \sum_{\infty}^{j-1} \mathrm{e}^{(\gamma-\beta) i}\right] \\
& <\sqrt{A} .
\end{aligned}
$$

In a similar way we obtain

$$
\frac{\left|\left(h_{\bar{\mu}}^{j}\right)^{\prime}(x)\right|}{\left|\left(h_{\bar{\mu}}^{j}\right)^{\prime}(\bar{\eta})\right|}>\frac{1}{\sqrt{A}},
$$

and conclusion (1) of the lemma follows.

Integrating the first inequality in (3), we see that there is a constant $\widetilde{K}, 0<\widetilde{K}<1$, such that $\left|h^{k+1}(\bar{\eta})-\bar{v}\right|>\widetilde{K}\left|h^{k}(\bar{\eta})\right|^{\alpha_{\mu, v}}$. Now, applying the mean value theorem to $h^{j}$, conclusion (1) and the hyphotesis of lemma, we obtain

$$
\left|h^{k+1+j}(\bar{\eta})-h^{j}(\bar{v})\right|>A^{-1} \lambda_{1}^{j} \widetilde{K} \mathrm{e}^{-r \alpha_{\mu, v}}, \quad j<k .
$$

From the binding period definition, for $j \leqslant s$ and $j<k$ we have $\mathrm{e}^{-\beta \gamma} \geqslant A^{-1} \lambda_{1}^{j} \widetilde{K} \times$ $\mathrm{e}^{-r \alpha_{\mu, \nu}}$, which implies that

$$
j \leqslant \frac{r \alpha_{\mu, \nu}-\log \left(\widetilde{K} A^{-1}\right)}{\beta+\log \left(\lambda_{1}\right)} .
$$

Next, choosing $\gamma$ and $\beta$ such that $2 \gamma \alpha_{\mu, \nu}<\beta$ and fixing

$$
k_{1}>\frac{-2 \log \left(\widetilde{K} A^{-1}\right)}{\beta+\log \left(\lambda_{1}\right)-2 \gamma \alpha_{\mu, \nu}},
$$

then for $j$ and $k$ as in the lemma, we obtain

$$
j \leqslant \frac{r \alpha_{\mu, \nu}-\log \left(\widetilde{K} A^{-1}\right)}{\beta+\log \left(\lambda_{1}\right)}<\frac{\gamma k \alpha_{\mu, v}-\log \left(\widetilde{K} A^{-1}\right)}{\beta+\log \left(\lambda_{1}\right)}<\frac{k}{2} .
$$


If $s \geqslant k$ then the inequality above holds for $j=k-1$ but this implies $k-1<k / 2$, which is a contradiction. Hence, $s<k$ and we can replace $j=s$ in the inequality above, obtaining the right inequality

$$
s \leqslant \frac{r \alpha_{\mu, \nu}-\log \left(\widetilde{K} A^{-1}\right)}{\beta+\log \left(\lambda_{1}\right)} .
$$

To establish the left inequality of conclusion (2) of the lemma, let

$$
D=\max \left\{\left|h_{\bar{\mu}}^{\prime}(x)\right|: p_{n}-\varepsilon \leqslant x \leqslant q_{n}+\varepsilon, \bar{\mu}\right\}>1 .
$$

Applying the mean value theorem and the binding period definition once again, we obtain a point $\xi \in\left(0, h_{\bar{\mu}}^{k}(\bar{\eta})\right)$ such that:

$$
D^{s+1}\left|h_{\bar{\mu}}^{k}(\bar{\eta})\right| \geqslant\left|\left(h_{\bar{\mu}}^{s+1}\right)^{\prime}(\xi)\right|\left|h_{\bar{\mu}}^{k}(\bar{\eta})-0\right|=\left|h_{\bar{\mu}}^{s+1}\left(h_{\bar{\mu}}^{k}(\bar{\eta})\right)-h_{\bar{\mu}}^{s}(\bar{v})\right|>\mathrm{e}^{-(s+1) \beta}
$$

wich implies $-(s+1) \beta<(s+1) \log (D)-r$, and the left inequality follows.

In order to obtain conclusion (3), let put

$$
t=\frac{\alpha_{\mu, \nu}}{\alpha_{\mu, \nu}-1}=1+\frac{1}{\alpha_{\mu, v}-1}
$$

and take $\xi \in\left(\bar{\nu}, h_{\bar{\mu}}^{k+1}(\bar{\eta})\right)$ such that

$$
\left(h_{\bar{\mu}}^{s}\right)^{\prime}(\xi)=\frac{\left|h_{\bar{\mu}}^{s+k+1}(\bar{\eta})-h_{\bar{\mu}}^{s}(\bar{\nu})\right|}{\left|h_{\bar{\mu}}^{k+1}(\bar{\eta})-\bar{v}\right|} .
$$

Note that $\left|\left(h_{\bar{\mu}}^{s}\right)^{\prime}\left(h_{\bar{\mu}}^{k+1}(\bar{\eta})\right)\right|>A^{-1}\left|\left(h_{\bar{\mu}}^{s}\right)^{\prime}(\xi)\right|$ implies

$$
\left|\left(h_{\bar{\mu}}^{s}\right)^{\prime}\left(h_{\bar{\mu}}^{k+1}(\bar{\eta})\right)\right|>A^{-1}\left|\left(h_{\bar{\mu}}^{s}\right)^{\prime}(\bar{v})\right|>A^{-1} \lambda_{1}^{s}
$$

(since $s<k$ ). Then

$$
\begin{aligned}
\left|\left(h_{\bar{\mu}}^{s+1}\right)^{\prime}\left(h_{\bar{\mu}}^{k}(\bar{\eta})\right)\right|^{t} & =\left|\left(h_{\bar{\mu}}^{s}\right)^{\prime}\left(h_{\bar{\mu}}^{k+1}(\bar{\eta})\right)\right|^{t}\left|h_{\bar{\mu}}^{\prime}\left(h_{\bar{\mu}}^{k}(\bar{\eta})\right)\right|^{t} \\
& \geqslant\left|\left(h_{\bar{\mu}}^{s}\right)^{\prime}\left(h_{\bar{\mu}}^{k+1}(\bar{\eta})\right)\right|\left|\left(h_{\bar{\mu}}^{s}\right)^{\prime}\left(h_{\bar{\mu}}^{k+1}(\bar{\eta})\right)\right|^{1 /\left(\alpha_{\mu, \nu}-1\right)} K_{1}^{t}\left|h_{\bar{\mu}}^{k}(\bar{\eta})\right|^{\alpha_{\mu, \nu}} \\
& \geqslant A^{-1}\left|\left(h_{\bar{\mu}}^{s}\right)^{\prime}(\xi)\right|\left|\left(h_{\bar{\mu}}^{s}\right)^{\prime}\left(h_{\bar{\mu}}^{k+1}(\bar{\eta})\right)\right|^{1 /\left(\alpha_{\mu, v}-1\right)} K_{1}^{t} \widetilde{K}\left|h_{\bar{\mu}}^{k+1}(\bar{\eta})-\bar{v}\right| \\
& >A^{-1} K_{1}^{t} \widetilde{K}\left|h_{\bar{\mu}}^{s+k+1}(\bar{\eta})-\bar{v}\right|\left|\left(h_{\bar{\mu}}^{s}\right)^{\prime}\left(h_{\bar{\mu}}^{k+1}(\bar{\eta})\right)\right|^{1 /\left(\alpha_{\mu, \nu}-1\right)} \\
& >A^{-1} K_{1}^{t} \widetilde{K} \mathrm{e}^{-\beta(s+1)} A^{-1 /\left(\alpha_{\mu, \nu}-1\right)} \lambda_{1}^{s /\left(\alpha_{\mu, v}-1\right)} \\
& =A^{-t} K_{1}^{t} \widetilde{K} \mathrm{e}^{-\log \lambda_{1} /\left(\alpha_{\mu, \nu}-1\right)} \exp \left[\left(\frac{\log \lambda_{1}}{\alpha_{\mu, \nu}-1}-\beta\right)(s+1)\right] \\
& =\tau^{t} \exp \left[\left(\frac{\log \lambda_{1}}{\alpha_{\mu, v}-1}-\beta\right)(s+1)\right] .
\end{aligned}
$$


Finally, if $\beta$ is small the coeficient of $(s+1)$ in the exponential above is positive, and the inequality in conclusion (3) of lemma follows for $s$ large, which is obtained making $\delta$ small (compatible with the previous conditions imposed on $\alpha$ and $\beta$ ).

A free return index is a return index that does not belong to a binding period associated to a previous return. Take $\gamma, \beta$ and $\delta$ as in Lemma 5, and for any positive integer $k$ let consider $i_{1}<i_{2}<\cdots<i_{l} \leqslant k$ all free return indices, i.e., $\mathrm{e}^{-\gamma i_{t}}<$ $\left|h_{\bar{\mu}}^{i_{t}}(\bar{v})\right| \leqslant \delta$, and let $s_{1}, s_{2}, \ldots, s_{l-1},\left(s_{l}\right)$ be the binding periods associated to returns $h_{\bar{\mu}}^{i_{1}}(\bar{v}), \ldots, h_{\bar{\mu}}^{i_{l-1}}(\bar{v}), h_{\bar{\mu}}^{i_{l}}(\bar{v})$. Note that $s_{l}$ will be or will be not considered according to $i_{l}<i_{l}+s_{l} \leqslant k$ or $i_{l} \leqslant k<i_{l}+s_{l}$. We say that $j, j \leqslant k$ is a free time for the critical value $\bar{v}$ asociated to the parameter $(\bar{\mu}, \bar{v})$ if $\left|h_{\bar{\mu}}^{j}(\bar{v})\right|>\delta$ and $j \notin\left[i_{t}, i_{t}+s_{t}\right], t=1, \ldots, l-1,(l)$. In the same way we define free time for the critical value $\bar{\mu}$ associated to the parameter $(\bar{\mu}, \bar{v})$.

Now, for $\bar{\eta}=\bar{\mu}$ or $\bar{\eta}=\bar{v}$ denote by $F(\bar{\eta}, k)$ the number given by:

$$
F(\bar{\eta}, k)=\#\{j \leqslant k: j \text { is a free time for the critical value }
$$

$\bar{\eta}$ asociated to the parameter $(\bar{\mu}, \bar{v})\}$.

Finally, fix $\varepsilon>0$ and define $\tilde{E}_{n, \theta}=\tilde{E}_{n, \theta}(\gamma, \beta, \delta, \varepsilon)$ by

$$
\tilde{E}_{n, \theta}=\left\{(\bar{\mu}, \bar{v}) \in \tilde{E}_{n, \theta}(\gamma): \min \{F(\bar{\mu}, k), F(\bar{v}, k)\} \geqslant(1-\varepsilon) k, k \in \mathbb{N}\right\} .
$$

Indeed, $\tilde{E}_{n, \theta}$ is the set of parameter values $(\bar{\mu}, \bar{v}) \in L_{n, \theta}$ satisfying the basic assumption (BA) and, in addition, the number of free times for the critical values $\bar{\nu}, \bar{\mu}$ associated to the parameter $(\bar{\mu}, \bar{v})$ is "almost" $k$, in fact bigger than $(1-\varepsilon) k$, for any $k \in \mathbb{N}$. Note that this last condition, called free time assumption (FA) implies $\sum_{t=1}^{l} s_{t}<\varepsilon k$.

As we have seen in the proof of Lemma 5 , by taking $\delta$ small we can increase the binding periods and we can assume $s_{t}>K_{0}, K_{0}$ as in Proposition 2.

The set of free times is the union of the free intervals $V_{1}=\left[1, \ldots, i_{1}-1\right], V_{2}=$ $\left[i_{1}+s_{1}+1, \ldots, i_{2}-1\right], \ldots, V_{l}=\left[i_{l-1}+s_{l-1}+1, i_{l}-1\right],\left(V_{l+1}=\left[i_{l}+s_{l}+1, k\right]\right)$. Denote $B$ as the set of indices $t$ such that $\# V_{t}<K_{0}$ and $G$ as the set of indices $t$ such that $\# V_{t} \geqslant K_{0}$. We have

$$
\begin{aligned}
& \# B<\sum_{t \in B} \# V_{t}<\sum_{t \in B} s_{t}<\sum_{t} s_{t}<\varepsilon k, \quad \text { and } \\
& \sum_{t \in G} \# V_{t} \geqslant(1-2 \varepsilon) k .
\end{aligned}
$$

Proposition 3. - For $\gamma, \beta, \delta$ and $\varepsilon$ small enough there are $\lambda, 1<\lambda<\lambda_{0}\left(\lambda_{0}\right.$ as in Proposition 2), $N \in \mathbb{N}$ and $a>0$ such that: for $n \geqslant N$ and $\left|(\bar{\mu}, \bar{v})-\left(\bar{\mu}_{n}, \bar{v}_{n}\right)\right|<a$, if $(\bar{\mu}, \bar{v}) \in \tilde{E}_{n, \theta}$ then $\left|\left(h_{\bar{\mu}}^{k}\right)^{\prime}(\bar{\mu})\right|>\lambda^{k}$ and $\left|\left(h_{\bar{\mu}}^{k}\right)^{\prime}(\bar{v})\right|>\lambda^{k}, k=1,2, \ldots$

Proof. - To start with, we proceed by induction on $k$. Take $1<\lambda<\lambda_{0}$ such that $\left|h_{\bar{\mu}_{\infty}, \bar{\nu}_{\infty}}^{\prime}(p)\right|>\lambda$ (here $p$ is the orientation preserving fixed point of $h_{\bar{\mu}_{\infty}, \bar{v}_{\infty}}$ ). Because $h_{\bar{\mu}}=h_{n, \bar{\mu}, \bar{v}}$ converges uniformly to $h_{\bar{\mu}, \bar{v}}$ we can find $N \in \mathbb{N}$ and $a>0$ such that for $n \geqslant N$ and $(\bar{\mu}, \bar{v})$ with $\left|(\bar{\mu}, \bar{v})-\left(\bar{\mu}_{n}, \bar{v}_{n}\right)\right|<a$ then $\left|\left(h_{\bar{\mu}}^{k}\right)^{\prime}(\bar{\eta})\right|>\lambda^{k}, \bar{\eta}=\bar{\mu}, \bar{v}, k \leqslant k_{1}$, where $k_{1}$ is taken as in Lemma 5 . And then, the first step in the induction works for 
$k \leqslant k_{1}$. Next, take $k>k_{1}$ and assume that the conclusion of the proposition is true for any iterate of $\bar{\eta}=\bar{\mu}, \bar{v}$ less than $k$. Because $k>k_{1}$ and $(\bar{\mu}, \bar{v})$ satisfies the basic assumption (BA), Lemma 5 works and it implies that during the binding periods there is not lost of derivative, in fact, the total derivative during those periods is bigger than 1 . From Proposition 2, it follows that during the free time intervals of length less than $K_{0}$ $(t \in B)$ we have a decreasing in the derivative of at most $C$ ( $C$ as in Proposition 2), whereas during the free time intervals of length bigger or equal than $K_{0}(t \in G)$ we have an increasing in the derivative of at least $\lambda_{0}^{\# V_{t}}$. Denote $C=\mathrm{e}^{-u}$. We can write $k=\sum_{t \in B} \# V_{t}+\sum_{t \in G} \# V_{t}+\sum_{t} \sum s_{t}$ and from the discusion above and (9) we obtain

$$
\begin{aligned}
\left|\left(h_{\bar{\mu}}^{k}\right)^{\prime}(\bar{\eta})\right| & \geqslant \prod_{t \in B} C \prod_{t \in G} \lambda_{0}^{\# V_{t}} \prod_{t=1}^{l} 1>\mathrm{e}^{-u \cdot \# B} \lambda_{0}^{\Sigma_{t \in G}} \# V_{t} \\
& >\mathrm{e}^{-u \varepsilon k} \lambda_{0}^{(1-2 \varepsilon) k}=\exp \left(\left[\log \lambda_{0}(1-2 \varepsilon)-u \varepsilon\right] k\right) .
\end{aligned}
$$

Taking $\varepsilon$ small we obtain $\log \lambda<\log \lambda_{0}(1-2 \varepsilon)-u \varepsilon$ (this choice works for any $k$ ) and then $\left|\left(h_{\bar{\mu}}^{k}\right)^{\prime}(\bar{\eta})\right| \geqslant \lambda^{k}$. This ends the proof of the proposition.

To finish up the proof of Theorem 2 we have to prove that it is possible to define a set $\tilde{E}_{n, \theta}$ as was mentioned before that satisfies:

(1) $m_{\theta}\left(\tilde{E}_{n, \theta}\right)>0$, where $m_{\theta}$ stands for the Lebesgue measure in $E_{n, \theta}$, and

(2) For $m_{\theta}$-almost every $(\bar{\mu}, \bar{v}) \in \tilde{E}_{n, \theta},{\overline{\left\{h_{\bar{\mu}}^{k}(\bar{\eta})\right\}_{k \in \mathbb{N}}}}=\left[h_{\bar{\mu}}(\bar{\xi}), \bar{\xi}\right]$, for $\bar{\eta} \in\{\bar{\mu}, \bar{v}\}$ where $\bar{\xi}=\max \{\bar{\mu}, \bar{v}\}$.

The set $\tilde{E}_{n, \theta}$ is constructed as in [2, Section 2]. This construction is nicely outlined in [10, Section 3], and here we refer to it. $\tilde{E}_{n, \theta}$ is obtained as the intersection of some sets $E_{k}$ in a small parameter interval having $\bar{\mu}_{n}$ in the right extreme, $E_{1} \supset E_{2} \supset \cdots \supset$ $E_{k} \supset \cdots$, satisfying the basic assumption (BA) and the free time assumption (FA). In order to define these sets $E_{k}$, some partition $\mathcal{P}_{k-1}$ of $E_{k-1}$ is required. The crucial point here is that the derivative of the maps $\bar{\mu} \rightarrow h_{\bar{\mu}}^{k}(\bar{\mu})$ and $\bar{\mu} \rightarrow h_{\bar{\mu}}^{k}(\bar{v})$ (remember that $\left.\bar{v}=\theta\left(\bar{\mu}-\bar{\mu}_{n}\right)+\bar{v}_{n}\right)$, require to have bounded distorsion on each interval $\omega$ in $\mathcal{P}_{k-1}([10$, Lemma 3.3] and [1, Lemma 5(7.2)]). This requirement and Lemma 5 enable us to estimate the Lebesgue measure of the set of points that will be excluded from $E_{k-1}$ to define $E_{k}$, all which is gotten as outlined in [10] (also see [15]). Thus, just following the same steps as in [10, Section 3], we obtain a set $\tilde{E}_{n, \theta}$ satisfying the basic assumption (BA), the free time assumption (FA) and $m_{\theta}\left(\tilde{E}_{n, \theta}\right)>0$.

Finally, in order to prove that the orbits of $\bar{\mu}$ and $\bar{v}$ are dense in $\left[h_{\bar{\mu}}(\bar{\xi}), \bar{\xi}\right]$ where $\bar{\xi}=\max \{\bar{\mu}, \bar{v}\}$ for almost every $(\bar{\mu}, \bar{v}) \in \tilde{E}_{n, \theta}$, we observe that the set of parameters such that the $h_{\bar{\mu}}$-orbit of $\bar{\mu}$ do not visit some fixed open interval is a zero Lebesgue measure set because $\bar{\mu} \rightarrow h_{\bar{\mu}}^{k}(\bar{\mu})$ is a distortion bounded map for all $k$ in the respective domain. The same conclusion holds for the critical value $\bar{v}$. Finally, using the fact that the topology of $L_{n, \theta}$ has a countable basis we can conclude that the set of parameters such that the critical values are not dense is a zero Lebesgue measure set. The proof of Theorem 2 is now complete. 


\section{REFERENCES}

[1] M. Benedicks, L. Carleson, On iterations of $1-a x^{2}$ on $(-1,1)$, Ann. Math. 122 (1985) $1-24$.

[2] M. Benedicks, L. Carleson, The dynamics of the Hénon map, Ann. Math. 133 (1991) 73169.

[3] R. Bamón, R. Labarca, R. Mañé, M.J. Pacífico, The explosion of singular cycles, Publ. Math. IHES 78 (1993) 207-232.

[4] P. Collet, J.P. Eckmann, On the abundance of aperiodic behaviour for maps on the interval, Comm. Phys. 73 (1980) 115-160.

[5] J. Guckenheimer, Sensitive dependence to initial conditions for one dynamical maps, Comm. Math. Phys. 70 (1979) 133-160.

[6] R. Labarca, Bifurcation of contracting singular cycles, Ann. Scient. Ec. Norm. Sup. 4e Série 28 (1995) 705-745.

[7] R. Labarca, M.J. Pacífico, Stability of singular horseshoe, Topology 25 (1986) 337-352.

[8] W. de Mello, S. van Strien, One-Dimensional Dynamics, in: Modern Surveys in Mathematics, Vol. 25, Springer-Verlag, Berlin, 1991.

[9] C. Morales, M.J. Pacifico, B. San Martín, Lorenz atractor from double homoclinic resonant cycles, Preprint.

[10] L. Mora, M. Viana, Abundance of strange attractors, Acta Math. 171 (1993) 1-71.

[11] M.J. Pacífico, A. Rovella, Unfolding contracting singular cycles, Ann. Scient. Éc. Norm. Sup. 4e Série 26 (1993) 691-700.

[12] J. Palis, On Morse-Smale dynamical systems, Topology 8 (1969).

[13] J. Palis, S. Smale, Structural Stability Theorems, in: Global Analysis, Proc. Symp. in Pure Math., Vol. XIV, American Mathematical Society, Providence, RI, 1970.

[14] J. Palis, F. Takens, Hyperbolicity \& Sensitive Chaotic Dynamics at Homoclinic Bifurcations, in: Cambridge Studies in Advanced Mathematics, Vol. 35, Cambridge University Press, Cambridge, 1993.

[15] A. Rovella, The dynamics of perturbations of the contracting Lorenz attractor, Bol. Soc. Bras. Mat. 24 (2) 233-259.

[16] B. San Martín, Contracting singular cycles, Ann. Inst. Henri Poincaré 15 (5) (1998) 651659.

[17] B. San Martín, J. Vera, Nonhyperbolic persistent attractors near the Morse-Smale boundary II, Informe de matemáticas UCN.

[18] M. Shub, Global Stability of Dynamical Systems, Springer-Verlag, Berlin, 1987. 